\title{
Tri-hamiltonian vector fields, spectral curves and separation coordinates
}

\author{
L. Degiovanni and G. Magnano \\ Dipartimento di Matematica, Università di Torino \\ Via Carlo Alberto 10, I-10131 Torino, Italy
}

\begin{abstract}
We show that for a class of dynamical systems, Hamiltonian with respect to three distinct Poisson brackets $\left(P_{0}, P_{1}, P_{2}\right)$, separation coordinates are provided by the common roots of a set of bivariate polynomials. These polynomials, which generalise those considered by E. Sklyanin in his algebro-geometric approach, are obtained from the knowledge of: (i) a common Casimir function for the two Poisson pencils $\left(P_{1}-\lambda P_{0}\right)$ and $\left(P_{2}-\mu P_{0}\right)$; (ii) a suitable set of vector fields, preserving $P_{0}$ but transversal to its symplectic leaves. The frameworks is applied to Lax equations with spectral parameter, for which not only it establishes a theoretical link between the separation techniques of Sklyanin and of Magri, but also provides a more efficient "inverse" procedure to obtain separation variables, not involving the extraction of roots.
\end{abstract}

\section{Introduction}

The relationship between the Liouville integrability of a Hamiltonian system and the existence of a second conserved Poisson bracket (or "hamiltonian structure") in its phase space, first discovered by Magri [1], has been thoroughly investigated in the past years. Bihamiltonian structures underlying all classical examples of integrable systems (both finite and infinite-dimensional) have been described by several authors, and almost all the relevant properties connected to integrability have been reinterpreted in terms of the geometry of bihamiltonian manifolds and vector fields. Recently, the classical problem of characterizing separable hamiltonians (i.e. those for which the Hamilton-Jacobi equation can be solved by separation of variables in a suitable system of canonical coordinates) has been also translated in the language of bihamiltonian geometry [2] [3].

A question which has not yet received a complete answer concerns the link between the bihamiltonian framework and the algebro-geometric methods of solution based on 
the isospectrality property of Lax equations [9] [8]. Although it is possible to introduce bihamiltonian structures which naturally lead to Lax equations with a spectral parameter [11] 21], the role of the characteristic equation for the Lax operator (the "spectral curve" of the algebro-geometric approach) has not been clarified so far in the bihamiltonian perspective.

The present work adds new elements in view of a connection between multihamiltonian structures, existence of separation coordinates and spectral curves, starting from an apparently marginal observation: some well-known integrable systems allow two distinct bihamiltonian descriptions, independently described by different authors and apparently unrelated (in spite of having one Poisson bracket in common). In this introductory section, we shall recall some relevant facts using the simplest example of such "trihamiltonian systems", namely the generalized Euler-Poinsot rigid body. To motivate the reader to follow us through an exercise which could seem of little practical interest, let us anticipate that the interplay of the three Poisson structures leads to a new role played by the characteristic determinant of the Lax matrix, and this fact may eventually clarify the connection between Sklyanin's algebro-geometrical construction of separation variables [12] and the bihamiltonian method recently proposed in [2] [3].

Indeed, the occurrence of more than two Poisson brackets on the same manifold is not new nor surprising by itself, and in some cases it is even a structural property, as for the so-called "Lie-Poisson pencils" described in [11]; in the sequel, we discuss the difference between such known cases of multihamiltonian structures and the trihamiltonian structure that we are presently considering.

The simplest (nontrivial) example of Lax equation with spectral parameter is provided by the dynamics of a rigid body about a fixed point, in the absence of external forces (Poinsot rigid body). In the body reference frame, the motion is described by the Euler-Poisson-Lax equation

$$
\frac{d M}{d t}=[M, \Omega]
$$

where $M$ and $\Omega$ are the skew-symmetric $3 \times 3$ matrices representing the angular momentum and the angular velocity, respectively, in the body reference frame. A straightforward consequence of (1.1) is that the trace of any power of the matrix $M$ is a constant of motion: $\frac{d}{d t} \operatorname{Tr}\left(M^{k}\right)=0$. Generalizing the system to $M \in \mathfrak{s o}(r)$, one obtains in this way at most $\frac{r}{2}$ independent constants of motion (if $r$ is even, or $\frac{r-1}{2}$ for $r$ odd), which for $r>3$ would not be enough to meet Liouville's integrability condition.

Assuming $\left(I_{1}, I_{2}, I_{3}\right)$ to be the eigenvalues of the inertia tensor, one can introduce the diagonal matrix with diagonal elements $\left(\frac{-I_{1}+I_{2}+I_{3}}{2}, \frac{I_{1}-I_{2}+I_{3}}{2}, \frac{I_{1}+I_{2}-I_{3}}{2}\right)$; the linear relation between $M$ and $\Omega$ can then be written in the following form:

$$
M=J \Omega+\Omega J
$$


Manakov [13] has observed that the Euler-Poisson equation (1.1) and the inertia map (1.2) can be put together into a single Lax equation for a new Lax pair dependent on a formal parameter $\lambda$,

$$
\frac{d\left(M+\lambda J^{2}\right)}{d t}=\left[M+\lambda J^{2}, \Omega+\lambda J\right] .
$$

The insertion of the parameter $\lambda$ into the Lax equation leads to a wider number of constants of motion, the Manakov integrals. We denote them by $f_{i}^{k}$, according to the following convention:

$$
f_{\lambda}^{(k)}=\frac{1}{k} J^{2 k} \lambda^{k}+\sum_{i=1}^{k} f_{i}^{k} \lambda^{k-i} .
$$

For $M \in \mathfrak{s o}(r)$, the functions $f_{i}^{k}(M)$ vanish identically for $i$ odd; the odd Manakov functions are however relevant for the "generalized Euler-Poinsot system", with $M \in$ $\mathfrak{g l}(r)$, that we shall consider in the sequel.

As is well known, the equation (1.1) is Hamiltonian with respect to the Lie-Poisson bracket, defined on $\mathfrak{s o}(r)$ through the ad-invariant scalar product

$$
(A, B)=\operatorname{Tr}(A \cdot B) .
$$

More precisely, given any function $f: \mathfrak{s o}(r) \rightarrow \mathbb{R}$, one defines the gradient at a point $M$ as the matrix $\nabla f \in \mathfrak{s o}(r)$ such that $\dot{f}=\langle d f, \dot{M}\rangle=(\dot{M}, \nabla f)$; then, for any pair of functions,

$$
\{f, g\}_{L P}=(M,[\nabla f, \nabla g])
$$

is a Poisson bracket [22]. The Lie-Poisson bracket (1.6) is degenerate: an ad-invariant function $f(M)$ is in involution with any other function, i.e. is a Casimir function for the bracket (1.6). The Casimir functions, which include the traces of the powers of $M$, are automatically constants of motion, but they are irrelevant as far as the Liouville integrability of the system (with the Lie-Poisson bracket) is concerned. Therefore, the integrability of the system actually relies on the existence of the other Manakov first integrals (among which one can find enough independent functions).

In 1996, Morosi and Pizzocchero [15 introduced a second Poisson bracket on $\mathfrak{s o}(r)$, defined as follows: let $A \in \mathfrak{g l}(r)$ a fixed matrix (for the Euler-Poinsot case, $A \equiv J^{2}$; notice that $A$ needs not to belong to $\mathfrak{s o}(r)$ ). With the same definition of scalar product and gradient as above, one sets

$$
\{f, g\}_{M P}=(M, \nabla f \cdot A \cdot \nabla g-\nabla g \cdot A \cdot \nabla f) .
$$

One can check that the vector field generated by the Manakov functions through the Poisson structure (1.7) are exactly the same as those generated through the Lie-Poisson structure (1.6), up to a rearrangement in the correspondence between hamiltonians and vector fields. For instance, the physical hamiltonian generating the Euler-Poinsot 
dynamics through the Lie-Poisson bracket is $h_{1}=\frac{1}{2} \operatorname{Tr}(\Omega M)=\operatorname{Tr}\left(\Omega^{2} J\right)$, while the hamiltonian of the same vector field through the Morosi-Pizzocchero bracket is $h_{2}=$ $-\frac{1}{2} \operatorname{Tr}\left(\Omega J^{-1} M J^{-1}\right)$. To simplify the notation, let us denote by $P_{1}$ and $P_{2}$ the Poisson tensors associated respectively to the brackets (1.6) and (1.7):

$$
\{f, g\}_{L P}=\left\langle d f, P_{1} d g\right\rangle, \quad\{f, g\}_{M P}=\left\langle d f, P_{2} d g\right\rangle .
$$

Denoting by $X_{1}$ the vector field over $\mathfrak{s o}(r)$ corresponding to equation (1.1), the relation $P_{1} d h_{1}=P_{2} d h_{2}$ is depicted by the diagram

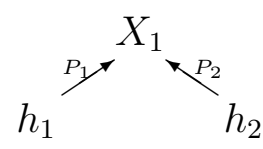

where $h \stackrel{P}{\rightarrow} X$ is an abbreviation for $d h \stackrel{P}{\longmapsto} X$, a convention that we shall use in analogous diagrams throughout this article. The diagram (1.9) is nothing but the elementary block of the Lenard-Magri recursion generating a whole family of quadratic first integrals $h_{i}$ (known as Miščenko functions), and the corresponding symmetry vector fields $X_{i}$ :

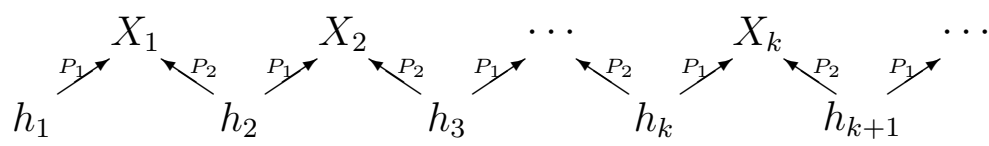

The Manakov first integrals can be generated by the same recursion procedure. Setting $A \equiv J^{2}$, one has $\nabla f_{\lambda}^{(k)}=(M+\lambda A)^{k-1}$, then

$$
P_{1} d f_{i}^{k}=\left[M, \nabla f_{i}^{k}\right]=A \nabla f_{i}^{k-1} M-M \nabla f_{i}^{k-1} A=P_{2} d f_{i}^{k-1}
$$

which correspond to Lenard-Magri diagrams starting with the $P_{1}$-Casimir functions $f_{k}^{k}$ :

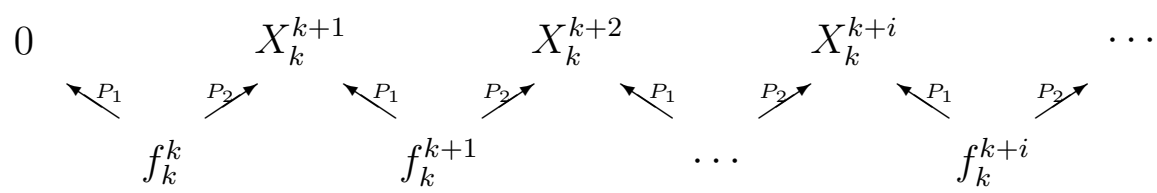

Notice that all the functions iteratively generated by Lenard-Magri recursion relations are automatically in involution with respect to both Poisson tensors $P_{1}$ and $P_{2}$. The (elementary) proof of this fact will be recalled in the next section. Thanks to this property of bihamiltonian vector fields, one does not need to prove separately the involutivity of the first integrals of Manakov, and the complete integrability of the generalized Euler-Poinsot system is simply assessed by computing how many independent first integrals can be found in this way. 
All the statements above hold valid if one extends the equation (1.1) to $M \in \mathfrak{g l}(r)$. Both the Lie-Poisson bracket and the Morosi-Pizzocchero bracket can be introduced in $\mathfrak{g l}(r)$ using the same definitions (1.6) and (1.7). The Morosi-Pizzocchero bracket is defined in terms of the matrix product (not of the commutator) and therefore is defined even more naturally on $\mathfrak{g l}(r)$ : it reduces on $\mathfrak{s o}(r)$ by orthogonal projection with respect to the scalar product (1.5), provided the matrix $A$ is symmetric. Thus, for the Lax matrix $L(\lambda)=A \lambda+M$ the complete family of Manakov constants of motion can be found by the recursion procedure, which also ensures their mutual involutivity. Whenever $A$ is symmetric (and positive), the dynamical system defined by (1.1) in $\mathfrak{g l}(r)$ is a proper extension of the original Euler-Poinsot system. The flows of the original model are those associated to the even Manakov functions (these flows are tangent to $\mathfrak{s o}(r)$ ), while the other flows of the enlarged system, generated by the odd Manakov functions, are orthogonal to $\mathfrak{s o}(r)$.

In the larger phase space $\mathfrak{g l}(r)$, however, one can obtain the full set of first integrals by another Lenard-Magri recursion, relative to a different bihamiltonian pair. The new Poisson bracket depends, as for (1.7), on the choice of the matrix $A$ :

$$
\{f, g\}_{A}=(A,[\nabla g, \nabla f]) \text {. }
$$

From now on, let us denote by $P_{0}$ the Poisson tensor associated with this bracket. The Manakov functions are in bihamiltonian recursion also with respect to the pair $\left(P_{0}, P_{1}\right)$, but the sequences are arranged in a different way:

$$
P_{1} d f_{i}^{k}=\left[M, \nabla f_{i}^{k}\right]=\left[\nabla f_{i+1}^{k}, A\right]=P_{0} d f_{i+1}^{k}
$$

Thus, each integer power of $L(\lambda)$ corresponds to a single finite Lenard-Magri sequence, starting from a Casimir function for $P_{0}$ and ending with a Casimir function for $P_{1}$ :

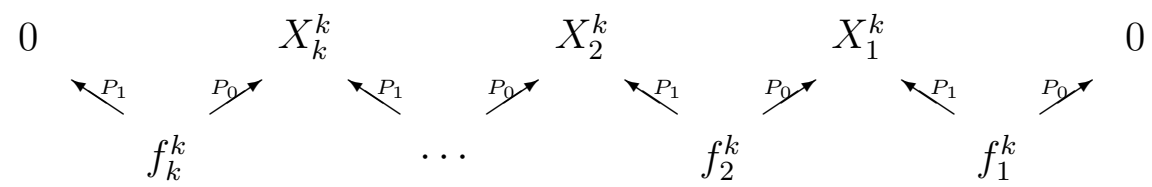

A disadvantage of the new bihamiltonian structure $\left(P_{0}, P_{1}\right)$ is that it cannot be reduced (by restriction or by orthogonal projection) to $\mathfrak{s o}(r)$. On the other hand, $\left(P_{0}, P_{1}\right)$ leads naturally to the Lax equation with spectral parameter (1.3), which on the contrary is rather difficult to derive from the former pair $\left(P_{1}, P_{2}\right)$. To show this, we need to reexpress the Lenard-Magri recursion relations (1.14) in the language of Poisson pencils.

Given a pair of Poisson tensors $(P, Q)$ on a manifold $\mathcal{M}$, assume that the $\lambda$ dependent bracket

$$
\{f, g\}_{P-\lambda Q}=\{f, g\}_{P}-\lambda\{f, g\}_{Q}=\langle d f,(P-\lambda Q) d g\rangle ;
$$


be a Poisson bracket, i.e. fulfill the Jacobi identity for any $\lambda$; in this case, $P$ and $Q$ are said to be compatible; $(\mathcal{M}, P, Q)$ becomes a bihamiltonian manifold (or $P Q$-manifold, following [4]), and one refers to the bracket (1.15) as to its Poisson pencil. It is immediate to see that, given a sequence of functions $\left\{f_{i}\right\}_{i=0, \ldots, N}$ such that

$$
\begin{aligned}
0 & =P d f_{0} \\
Q d f_{0} & =P d f_{1} \\
& \vdots \\
Q d f_{k} & =P d f_{k+1} \\
& \vdots \\
Q d f_{N} & =0,
\end{aligned}
$$

then the polynomial in $\lambda$ defined by

$$
f_{\lambda}=f_{0}+f_{1} \lambda+\cdots+f_{N} \lambda^{N}
$$

is a Casimir function of the Poisson pencil, i.e. for any $\lambda$

$$
(P-\lambda Q) d f_{\lambda}=0
$$

(the differential of $f_{\lambda}$ is taken with respect to the coordinates on $\mathcal{M}, \lambda$ being regarded as a parameter). Conversely, given a $\lambda$-polynomial function fulfilling (1.18), its coefficients obey the Magri-Lenard recursion according to (1.16) and generate a sequence of commuting bihamiltonian vector fields.

In the next section, we will recall the proof of the following relevant property, that we shall extensively use. Let $g_{\lambda}$ be a second Casimir function of the same Poisson pencil: then, not only its coefficients $g_{k}$ are in involution among themselves, but they also Poisson-commute with all the coefficients $f_{k}$ of the other Casimir function $f_{\lambda}$.

Given a polynomial Casimir function $f_{\lambda}$, each bihamiltonian vector field of the associated Lenard-Magri hierarchy $X_{k}=P d f_{k}=Q d f_{k-1}$ can be also represented by a Hamilton equation with spectral parameter. Having set $f_{\lambda}=\sum_{i=0}^{N} f_{i} \lambda^{i}$, consider for each positive integer $k<N$ the polynomial

$$
f_{\lambda}^{(k)} \equiv f_{0} \lambda^{k}+f_{1} \lambda^{k-1}+\cdots+f_{k-1} \lambda+f_{k} ;
$$

taking into account (1.16) it is easy to see that

$$
X_{k}=(P-\lambda Q) d f_{\lambda}^{(k)} .
$$

This formula holds true for formal power series $(N=\infty)$; if the Casimir function $f_{\lambda}$ is instead expanded in Laurent series, $f_{\lambda}=\sum_{i=0}^{\infty} f_{i} \lambda^{-i}$, then the polynomial $f_{\lambda}^{(k)}$ is easily obtained by multiplication by $\lambda^{k}$ and truncation to the nonnegative powers:

$$
f_{\lambda}^{(k)}=\left(\lambda^{k} f_{\lambda}\right)_{+} .
$$


We are now ready to derive the Manakov equation (1.3) as a Hamilton equation with spectral parameter for the Poisson pencil $\left(P_{1}-\lambda P_{0}\right)$ on $\mathfrak{g l}(r)$.

In fact, it is easy to see that the trace of any power of the Lax matrix $A \lambda+M$ is a Casimir function of the Poisson pencil $\left(P_{1}-\lambda P_{0}\right)$ : by definition (1.6, 1.12),

$$
\left(P_{1}-\lambda P_{0}\right) d f=[A \lambda+M, \nabla f]
$$

as already seen, for $f_{\lambda}^{(k)}=\frac{1}{k} \operatorname{Tr}(A \lambda+M)^{k}$ one has $\nabla f_{\lambda}^{(k)}=(A \lambda+M)^{k-1}$, which obviously commutes with $A \lambda+M$. The same happens for the Laurent series expansion of the trace of any half-integer power of $A+M \lambda^{-1}$. On account of (1.20), all the vector fields generated by the coefficients of these Casimir functions (which all mutually commute, by the property mentioned above) correspond to Lax equations with spectral parameter:

$$
X_{k}=\left(P_{1}-\lambda P_{0}\right) d f_{\lambda}^{(k)}=\left[A \lambda+M, \nabla f_{\lambda}^{(k)}\right] .
$$

In particular, the Manakov equation (1.3) corresponds to the first vector field of the hierarchy associated to the Casimir function $f_{\lambda}=\frac{2}{3} \operatorname{Tr}\left(A+M \lambda^{-1}\right)^{3 / 2}$, for which $\nabla f_{\lambda}^{(1)}=$ $A^{1 / 2} \lambda+\Omega$. No comparably simple and natural connection exists between the other Poisson pencil $\left(P_{2}-\lambda P_{1}\right)$ and the Lax-Manakov form of the equations. This setting can be generalized to cover the cases of more general Lax matrices with spectral parameter on $\mathfrak{g l}(r)$, of the form $L(\lambda)=A \lambda^{k}+M_{1} \lambda^{k-1}+\cdots+M_{k}$. The general framework is described in [11], and will be partially recalled in section (4) below.

So far, we have simply reviewed some results already present in the literature. Now, some questions arise naturally. We have described a dynamical system which is bihamiltonian with respect to two independent PQ structures, $\left(P_{0}, P_{1}\right)$ and $\left(P_{1}, P_{2}\right)$; is that a pure accident, or it is a common situation?

One can check by explicit computation that the Poisson tensors $P_{0}$ and $P_{2}$ are not only separately compatible with $P_{1}$, but also compatible with each other (that is not obvious, as compatibility is not a transitive relation). Does it make any sense to introduce the notion of a trihamiltonian structure $\left(P_{0}, P_{1}, P_{2}\right)$ ? Would it carry any additional information not already contained in either one of the PQ structures, each of which already allows to characterize completely the dynamical system and its symmetries?

The vector fields (1.23) on $\mathfrak{g l}(r)$ are indeed trihamiltonian. The full set of hamiltonians and vector fields generated by the traces of integers powers of $A \lambda+M$, fit into a single "planar" diagram (as was first pointed out by M. Ugaglia [14]), which could be 
regarded as the "trihamiltonian version" of the Lenard-Magri "linear" diagrams (1.14):

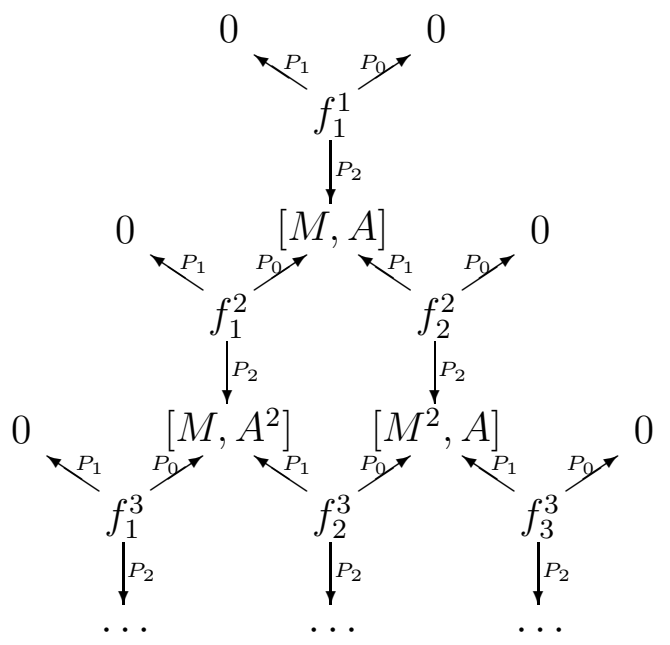

We have seen above that, for a $\mathrm{PQ}$ structure, any linear recursion starting from a Casimir function of $Q$ and ending with a Casimir function of $P$ corresponds to the existence of a $\lambda$-polynomial Casimir function of the Poisson pencil $(P-\lambda Q)$. Can one find a "generating polynomial" for the full trihamiltonian recursion? The answer is yes: as we shall see in detail in section (2), if one considers two compatible Poisson pencils $\left(P_{1}-\lambda P_{0}\right)$ and $\left(P_{2}-\mu P_{0}\right)$, one can define a common Casimir function of the two pencils to be a bivariate polynomial $f_{\lambda \mu}=\sum h_{j}^{i} \lambda^{j} \mu^{i}$ such that

$$
\begin{aligned}
& \left(P_{1}-\lambda P_{0}\right) d f_{\lambda \mu}=0 \\
& \left(P_{2}-\mu P_{0}\right) d f_{\lambda \mu}=0
\end{aligned}
$$

for any value of $(\lambda, \mu)$ : then its coefficients $h_{j}^{i}$ fulfill the recursion relations represented in the diagram (1.24). Later on we will explain why the construction of two Poisson pencils, each one with its own spectral parameter, is here more fruitful than introducing a two-parameter pencil like $\left(P_{0}-\lambda P_{1}-\mu P_{2}\right)$.

Up to this point, the reader might still regard the idea of trihamiltonian structures as an artifact of purely academic interest, a mere "variation on the theme" of bihamiltonian structures. Two results, presented in this article, suggest that the subject is worth investigating further.

First, the trihamiltonian structure associate to equation (1.3) can be generalized, in quite a nontrivial way, to Lax equations for matrices of the form $L(\lambda)=A \lambda^{n}+$ $M_{1} \lambda^{n-1}+\cdots+M_{n}$, which include several interesting systems such as the Lagrange top [17] and the finite-dimensional Dubrovin-Novikov reductions of the Gel'fand-Dickey soliton hierarchies [18]. Indeed, the generalization of the pencil $\left(P_{1}-\lambda P_{0}\right)$ to the direct sum of $n$ copies of $\mathfrak{g l}(r)$ was already described in [11], but to our knowledge it is an 
entirely new result that also the Morosi-Pizzocchero bracket is a particular case of a more general structure existing on $\mathfrak{g l}(r)^{n}$, a Poisson tensor $P_{2}$ which turns out to be quadratically dependent on the dynamical variables $M_{i}$, apart for the linear case $n=1$ already discussed.

The second striking fact is that for these trihamiltonian structures on $\mathfrak{g l}(r)^{n}$ there always exists a common Casimir function of the two pencils, which (for a generic choice of the matrix $A$ ) has the property that its coefficients form a maximal set of independent hamiltonians in involution (we stress that, in contrast, the recursion diagram for the traces of the powers of the Lax matrix includes infinitely many hamiltonians, and one has to single out a finite subset of independent first integrals). This miraculous Casimir function is nothing but the characteristic determinant of the Lax matrix,

$$
f_{\lambda \mu}=\operatorname{det}|L(\lambda)-\mu \mathbb{I}| \text {. }
$$

The corresponding recursion diagram features a sort of "fundamental molecule", a "fingerprint" associated to the trihamiltonian structure $\left(P, Q_{1}, Q_{2}\right)$. For instance, the following diagram corresponds to the trihamiltonian recursion on the algebra $\mathfrak{g l}(3)$ :

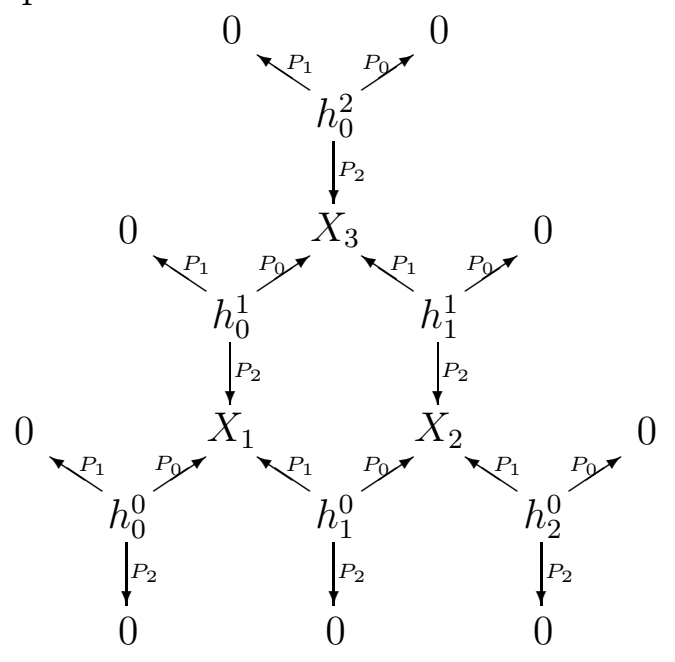

while $(\underline{1.28})$ is the "molecule" of the trihamiltonian structure on $\mathfrak{g l}(2)^{2}$ :

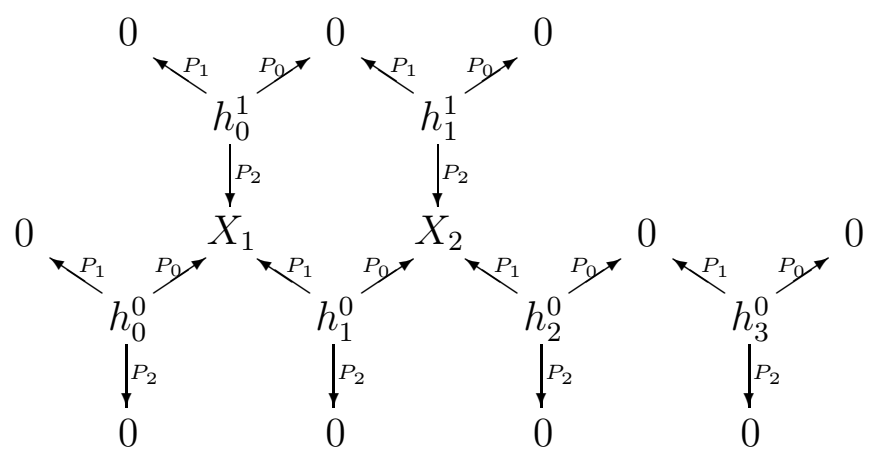


The general form of the "fundamental molecule" for $\mathfrak{g l}(r)^{n}$ is given in section (4) as fig.1.

Although it is a well known fact that the coefficients of the characteristic polynomial are in involution with respect to the usual Lie-Poisson bracket, in the bihamiltonian framework there was no apparent reason to introduce a bivariate polynomial $f_{\lambda \mu}$ in connection with the Lenard-Magri recursion. For a trihamiltonian structure, instead, it is quite natural to consider this object, and the characteristic polynomial of a Lax matrix becomes just a particular case of it, in exactly the same way as Lax equations with spectral parameter are a particular case of Hamilton equations, for the appropriate Poisson pencil (1.20).

This opens a very interesting perspective. The characteristic equation

$$
\operatorname{det}|L(\lambda)-\mu \mathbb{I}|=0
$$

regarded as a polynomial equation for $(\lambda, \mu) \in \mathbb{C}^{2}$ defines the well-known spectral curve, i.e. the starting point for the algebro-geometric methods of linearisation [9] [8]. In the trihamiltonian framework, as we have seen, the characteristic determinant naturally occurs as the fundamental Casimir function of two pencils: yet this does not explain why the roots $\left(\lambda_{i}, \mu_{i}\right)$ of eq. (1.29) should play any role at all. Now comes a third surprise: a fairly general construction presented in section (3) shows that the equation $f_{\lambda \mu}=0$ is the keystone for the construction of canonical separation coordinates for trihamiltonian systems.

This result essentially derives from an observation by E. Sklyanin [12]. On algebrogeometric grounds, Sklyanin has found a "magic recipe" ("Take the poles of the properly normalized Baker-Akhiezer function and the corresponding eigenvalues of the Lax operator"), which essentially amounts to finding the common roots of (1.29) and of suitable minors (or linear combination of minors) of the characteristic matrix $L(\lambda)-\mu \mathbb{I}$. In the examples considered by Sklyanin, the new variables $\left(\lambda_{i}, \mu_{i}\right)$ defined in this way turn out to be canonical with respect to a suitable Poisson bracket; by direct consequence of eq. (11.29), all the hamiltonians defined as the (nonconstant) coefficients of $f_{\lambda \mu}$ are then separable in the coordinates $\left(\lambda_{i}, \mu_{i}\right)$. However, Sklyanin himself remarks that "generally speaking, there is no guarantee that one obtains the canonical Poisson brackets [..] The key words in the above recipe are 'the properly normalized'. The choice of the proper normalization can be quite nontrivial, and for some integrable models the problem remains unsolved".

Independently of Sklyanin's approach, Magri and his collaborators [2] [3] have recently shown that given (i) a PQ structure, (ii) a complete family of commuting hamiltonians defined by the Casimir functions of the Poisson pencil, and (iii) a set of vector fields, suitably normalized on the hamiltonians previously introduced, which preserve the Poisson tensor $P$ but do not belong to its image (geometrically speaking, they should be transversal to the symplectic leaves of $P$ ), then one can define by projection (under 
some additional conditions on the vector fields) a reduced, kernel-free bihamiltonian structure; for this new PQ structure, a set of Darboux-Nijenhuis canonical coordinates can be obtained by a constructive procedure, and the original hamiltonians (properly reduced) turn out to be all simultaneously separable in these coordinates.

The theoretical interest of both constructions is largely beyond the concrete applicability of these procedures. As a matter of fact, while Sklyanin's recipe lacks a general, theoretically-grounded rule to find the key element (the normalization of the BA function, or equivalently the proper linear combination of minors of the Lax matrix which should vanish), in Magri's theory there is no practical recipe to construct systematically sets of transversal vector fields fulfilling the necessary requirements. In both approaches, moreover, the final construction of separation coordinates involves finding the roots of polynomial equations, which even for rather simple examples turn out to be of order higher than three.

As we show in this article, Magri's procedure can be adapted to the trihamiltonian setup, without loosing its geometric elegance, and actually making the theory even simpler and more symmetric (although less general). In this framework, the central role of the "generalized spectral equation" $f_{\lambda \mu}=0$ becomes clear. Moreover, for the trihamiltonian structures that we are introducing on the spaces $\mathfrak{g l}(r)^{n}$, we have found a systematic way to produce explicitly the required transversal vector fields, and we will show that the associated Darboux-Nijenhuis coordinates are exactly the roots of suitable combinations of minors of the characteristic matrix, much alike Sklyanin's coordinates; in this way, we provide for this class of systems the missing element in both Sklyanin's and Magri's prescriptions for the construction of separation variables. In addition, we show that our framework makes available a different strategy, which yields the inverse transformation (i.e. the matrix elements of the Lax operator as functions of the separation variables) by solving only a system of linear algebraic equations, thus bypassing the problem of finding roots of higher-order polynomials.

Let us quote another important remark by Sklyanin [12]: "Separation of variables, understood generally enough, could be the most universal tool to solve integrable models [...] the standard construction of the action-angle variables from the poles of the Baker-Akhiezer function can be interpreted as a variant of separation of variables, and moreover, for many particular models it has a direct quantum counterpart". Therefore, a satisfactory hamiltonian setup for Sklyanin's construction is likely to provide a link between hamiltonian and algebro-geometric integrability. In this sense, the equation $f_{\lambda \mu}=0$ should deserve some additional interest, as it points towards a generalisation of the notion of spectral curve not relying on Lax representations.

The article is organized as follows: in section (2) we recall, as synthetically as possible, some facts about bihamiltonian structures which are necessary for the subsequent discussion; then, we present a theoretical setting of our class of trihamiltonian structures. In section (3) we discuss the general method of construction of separation variables, i.e. the trihamiltonian version of Magri's construction. We present in detail the proofs 
of some relevant propositions providing the theoretical background for all applications of our framework; furthermore, we show how the components of all relevant objects (Poisson structures, common Casimir function, transversal vector fields, etc.) look like in Darboux-Nijenhuis coordinates; this will be used in section (4) to reconstruct the coordinate transformation. The fourth and last section is devoted to the application to Lax equations with spectral parameter on $\mathfrak{g l}(r)^{n}$; here we simply list the "ingredients of the recipe" without a general proof; this section is intended to present a concrete outcome in just enough detail to motivate the reader to deal with the theoretical construction of section (3).

Throughout the article no attempt is made to present a geometric characterisation, or classification, of the trihamiltonian structures possessing the specific features considered. In particular, we deliberately avoid to encompass these features into a single definition of "special trihamiltonian structure". In our discussion, the basic structure involved is sometimes presented as a triple of compatible Poisson tensors, but more often as a pair of Possion pencils; the assumptions on this basic structure vary according to the context. In sect.(2) we just reconsider the notion of "trihamiltonian recursion" associated with a common Casimir function of two Poisson pencils, without imposing particular conditions on the Poisson tensors besides their mutual compatibility; in such generality, indeed, nothing ensures that a common Casimir function exist at all (we will present a counterexample). In sect.(3.1) we show how to define a pair of Nijenhuis tensors using a set of vectorfields, which should fulfill a number of requirements: these Nijenhuis tensors are related with a particular system of Darboux-Nijenhuis coordinates, and do not act as recursion operators for the trihamiltonian iteration described previously (throughout this subsection, the existence of a common Casimir function is irrelevant). In section (3.2) the two objects, i.e. the common Casimir function (that we now require to be complete in a suitable sense) and the set of transversal vectorfields, are eventually combined together to construct a set of bivariate polynomials $S_{\alpha}(\lambda, \mu)$ : then, a number of additional hypotheses are introduced to obtain the main result, i.e. that the common roots of these polynomials are Darboux-Nijenhuis coordinates, in which the Hamiltonians occurring as coefficients in the common Casimir function are separated (in the sense of Sklyanin). Hence, the set of conditions to be imposed on the basic structure depends on whether one looks for the mere existence of iterated trihamiltonian vectorfields, or for a possible explicit construction of separation coordinates. In conclusion, we feel that at the present stage of understanding of the matter presented herein, one could hardly find a simple and general definition which might be regarded as truly fundamental. The aim of the article is rather to display a number of nontrivial and rigorous (if not yet complete) arguments in favour of further investigations in this direction. 


\section{From bi- to trihamiltonian structures}

\subsection{Poisson pencils}

As was already done (1.8) in the introductory section, we shall represent a Poisson bracket $\{\cdot, \cdot\}$ on a manifold $\mathcal{M}$ by means of a contravariant antisymmetric tensorfield $P$, according to

$$
\langle d g, P d f\rangle=\{f, g\} .
$$

The names Poisson structure or hamiltonian structure are equivalently used, as is commonly done, to denote both the tensor $P$ and the algebra of differentiable functions on $\mathcal{M}$ with the bilinear operation defined by the corresponding Poisson bracket. Of course, a contravariant antisymmetric tensorfield $P$ defines a hamiltonian structure only if the bracket (2.1) obeys the Jacobi identity; this condition corresponds to a differential identity on the components of $P$.

In most of our applications, the tensor $P$ will not be of maximal rank; thus, the subalgebra of functions which are in involution with any other function may include non-constant functions, the Casimir functions. The Casimir functions are constant of motion for any hamiltonian vectorfield, i.e. for any vectorfield being the image of a closed one-form through the Poisson tensor $P$. Therefore, any trajectory of any possible hamiltonian system on that phase space lies entirely on a common level set of all the Casimir functions. Generically, such a level set is a submanifold, the dimension of which equals the rank of the Poisson tensor. Upon reduction to any of these submanifolds, the Poisson tensor becomes invertible and therefore defines a symplectic structure. For this reason, the common level sets (for regular values) of the Casimir functions are called symplectic leaves. In contrast with the case of symplectic manifolds, the Lie derivative the Poisson tensor can vanish along the flow of a given vectorfield $X$,

$$
\mathcal{L}_{X}(P)=0
$$

without $X$ being even locally hamiltonian: the condition (2.2) can be fulfilled also by vectorfields which do not belong to the image of $P$, and are therefore transversal to the symplectic leaves. Such vectorfields will be called weakly hamiltonian. They play an important role in the sequel.

Bihamiltonian structures were first introduced by Magri in [1].

Defininition 2.1: Two Poisson tensors $P$ and $Q$ on a manifold $\mathcal{M}$ are told to be compatible if any linear combination of the two tensors is again a Poisson tensor.

In such situation one can find vectorfields which are hamiltonian with respect to both structures, i.e. bihamiltonian vectorfields. In this article, we borrow from the bihamiltonian theory the following facts: 
(i) Two hamiltonians are associated to a single bihamiltonian vectors field $X=$ $P d h=Q d k$. Then, one can define two other vectorfields, namely $Q d h$ and $P d k$. In some cases, these turn out to be bihamiltonian as well, and the procedure can be iterated yielding a Magri-Lenard hierarchy of bihamiltonian vectorfields, as in (1.10).

(ii) Once a Magri-Lenard hierarchy has been constructed, all the vectorfields belonging to it are mutually commuting, and all their hamiltonians are in involution with respect to both $P$ and $Q$.

(iii) There are basically two ways to produce such hierarchies: if at least one of the Poisson tensors (say, $P$ ) is nondegenerate, then one can introduce the recursion operator (or Nijenhuis tensor)

$$
N=Q \cdot P^{-1}
$$

One can prove 44 that for any bihamiltonian vectors field $X$, the vectors field $N X$ is also bihamiltonian, so the hierarchy can be produced by iterated application of the $(1,1)$ tensors field $N$. Alternatively (for instance, if both Poisson tensors are degenerate), one can look for Casimir functions of the Poisson pencil $(Q-\lambda P)$, as already described in the Introduction.

The classical proof of the involutivity property, which is the most relevant to our purposes, is so simple and elegant that we reproduce it here (further details can be found in [6], [10]):

Proposition 2.1: Let $f_{\lambda}$ and $g_{\lambda}$ be two Casimir functions of the Poisson pencil $(Q-\lambda P)$. Assume that $f_{\lambda}$ and $g_{\lambda}$ are expanded in power series in the parameter $\lambda$ of the pencil, $f_{\lambda}=\sum_{i=0} f_{i} \lambda^{i}$ and $g_{\lambda}=\sum_{i=0} g_{i} \lambda^{i}$. Then $\left\{f_{j}, f_{k}\right\}=\left\{g_{j}, g_{k}\right\}=\left\{f_{j}, g_{k}\right\}=0$ for all $j, k$ : this holds for both brackets $\{,\}_{P}$ and $\{,\}_{Q}$ associate to $P$ and to $Q$ respectively.

Proof: The conditions $(Q-\lambda P) d f_{\lambda}=0$ and $(Q-\lambda P) d g_{\lambda}=0$ are equivalent to $P d f_{i}=Q d f_{i+1}$ and $P d g_{i}=Q d g_{i+1}$. Moreover, one should have $Q d f_{0}=0$ and $Q d g_{0}=0$, i.e. the lowest-order coefficients of both expansions should be Casimir function for $Q$. One can assume $j<k$, without loss of generality. From the definition (2.1) $\left\{f_{j}, f_{k}\right\}_{P}=$ $\left\{f_{j+1}, f_{k}\right\}_{Q}=\left\{f_{j+1}, f_{k-1}\right\}_{P}$. Whenever $k-j$ is even, applying repeatedly the equality one finds that $\left\{f_{j}, f_{k}\right\}_{P}=\left\{f_{r}, f_{r}\right\}_{P}=0$, with $r=(k-j) / 2$; otherwise, after $(k-j)$ steps one finds $\left\{f_{j}, f_{k}\right\}_{P}=\left\{f_{k}, f_{j}\right\}_{P}$, which proves the statement by the antisymmetry of the Poisson bracket. The same holds for $\left\{g_{j}, g_{k}\right\}_{P}$, and for the other bracket $\{,\}_{Q}$. Furthermore, applying the same iterative argument one finds $\left\{f_{j}, g_{k}\right\}_{Q}=\left\{f_{j+k}, g_{0}\right\}_{Q}$, and the latter bracket vanishes because $g_{0}$ is a Casimir function for $Q$. This proves that $\left\{f_{j}, g_{k}\right\}_{Q}=0$ for all $j, k$. Since $\left\{f_{j}, g_{k}\right\}_{P}=\left\{f_{j+1}, g_{k}\right\}_{Q}$, one has $\left\{f_{j}, g_{k}\right\}_{P}=0$ as well. 


\subsection{Casimir functions and trihamiltonian vector fields}

Assume that a manifold $\mathcal{M}$ is endowed with three Poisson tensors $P_{0}, P_{1}$ and $P_{2}$, pairwise compatible. A natural question is whether a set of vectorfields that are hamiltonian with respect to all three structures can be generated by the coefficients of some "generating function", analogous to the Casimir function $f_{\lambda}$ above, and whether the corresponding hamiltonians would then be automatically in involution.

One might believe that the obvious generalization of the setting just described would consist in introducing a two-parameter Poisson pencil

$$
P_{0}-\lambda P_{1}-\mu P_{2}
$$

and seeking for its Casimir functions. Unfortunately, the coefficients of the Taylor series in the two parameters $(\lambda, \mu)$ do not fit into any useful recursion relation: from the Casimir equation

$$
\left(P_{0}-\lambda P_{1}-\mu P_{2}\right) d f_{\lambda \mu}=0 \quad \text { for } \quad f_{\lambda, \mu}=\sum_{i, j=0}^{\infty} h_{i}^{j} \lambda^{i} \mu^{j}
$$

one gets the relations

$$
\begin{array}{rlrl}
P_{0} d h_{0}^{0} & =0 & \\
P_{0} d h_{i+1}^{0} & =P_{1} d h_{i}^{0} & i=0,1, \ldots \\
P_{0} d h_{0}^{j+1} & =P_{2} d h_{0}^{j} & j=0,1, \ldots \\
P_{0} d h_{i+1}^{j+1} & =P_{1} d h_{i}^{j+1}+P_{2} d h_{i+1}^{j} & i, j=0,1, \ldots
\end{array}
$$

which neither provide trihamiltonian vector fields nor force the functions $f_{i}^{j}$ to be in involution.

Let us consider instead a function $f_{\lambda \mu}$ that is simultaneously a Casimir function of the two distinct pencils $\left(P_{1}-\lambda P_{0}\right)$ and $\left(P_{2}-\mu P_{0}\right)$ :

$$
\left(P_{1}-\lambda P_{0}\right) d f_{\lambda \mu}=0, \quad\left(P_{2}-\mu P_{0}\right) d f_{\lambda \mu}=0 .
$$

In this case we actually obtain the following relations:

$$
\begin{aligned}
P_{1} d h_{0}^{j} & =0 & & j=0,1, \ldots \\
P_{0} d h_{i}^{j} & =P_{1} d h_{i+1}^{j} & & i, j=0,1, \ldots \\
P_{2} d h_{i}^{0} & =0 & & i=0,1, \ldots \\
P_{0} d h_{i}^{j} & =P_{2} d h_{i}^{j+1} & & i, j=0,1, \ldots
\end{aligned}
$$


graphically:

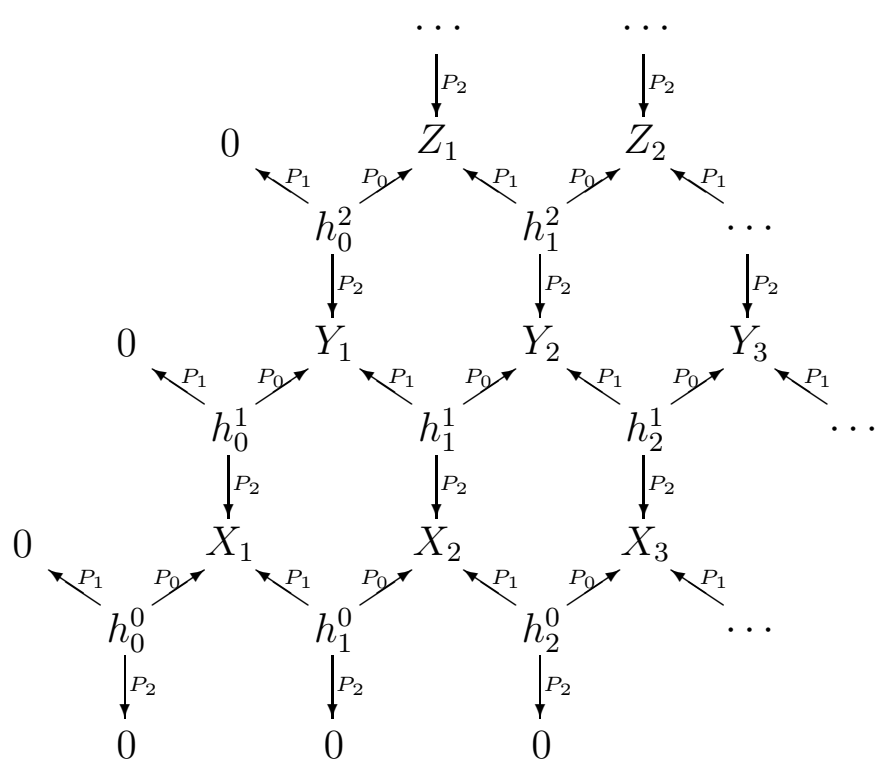

the vectorfields $P_{0} d h_{i}^{j}=P_{1} d h_{i+1}^{j}=P_{2} d h_{i}^{j+1}$ are clearly trihamiltonian.

Notice that it is possible to find a common Casimir function which can be (formally) expanded in a Taylor series with respect to the two parameters $\lambda$ e $\mu$ only if both $P_{1}$ and $P_{2}$ are degenerated Poisson tensors: in fact, for a fixed power of $\lambda$, the lowest-order coefficient in $\mu$ must be a Casimir function of $P_{2}$, while the lowest-order coefficient in $\lambda$ for any fixed power of $\mu$ must be a Casimir function of $P_{1}$. If, moreover, also $P_{0}$ is degenerate, then it is possible to find Casimir functions which are polynomials in $\lambda$ and $\mu$. For such functions the recursion diagram is finite.

In analogy with the bihamiltonian case, one has:

Proposition 2.2: Given a common Casimir function $f_{\lambda \mu}=\sum h_{i}^{j} \lambda^{i} \mu^{j}$ of two compatible Poisson pencils $P_{1}-\lambda P_{0}$ and $P_{2}-\mu P_{0}$, all the coefficients $h_{i}^{j}$ are in mutual involution with respect to all three Poisson brackets.

Proof: For any $i, j$ the functions $h_{\lambda}^{(i)}=\sum h_{k}^{i} \lambda^{k}$ and $h_{\lambda}^{(j)}=\sum h_{k}^{j} \lambda^{k}$, i.e. the total coefficients of $\mu^{i}$ and $\mu^{j}$ in the expansion of $f_{\lambda \mu}$, are Casimir functions of the Poisson pencil $\left(P_{1}-\lambda P_{0}\right)$; then, by proposition (2.1) all their coefficients $h_{i}^{j}$ are in involution with respect to both $P_{0}$ and $P_{1}$. On the other hand, the functions $h_{\mu}^{(i)}=\sum h_{i}^{k} \mu^{k}$ and $h_{\mu}^{(j)}=\sum h_{j}^{k} \mu^{k}$ are Casimir functions of the other pencil $P_{2}-\mu P_{0}$, hence $h_{i}^{j}$ are in involution also with respect to $P_{2}$.

We remark that there are other possible ways to extend the bihamiltonian framework to the case in which there are more than two compatible Poisson structures. The 
idea of a trihamiltonian vector field was already considered, for example, in [19 and [16]), where the three structures $P, Q$ and $S$ were however assumed to produce the iteration $P \mathrm{~d} h_{i}=Q \mathrm{~d} h_{i+1}=S \mathrm{~d} h_{i-1}$. In this approach, the third structure only supplies an additional relation which links vectorfields already belonging to the same MagriLenard hierarchy; in our framework, the third structure acts instead as a bridge linking different bihamiltonian hierarchies, and so allows to collect a greater number of function in involution in a single objet: the common Casimir function.

In our setup, the structure $P_{0}$ seem to play a distinguished role with respect to $P_{1}$ and $P_{2}$. As a matter of fact, it is easy to figure out how to include in the picture also the Poisson pencil built from $P_{1}$ and $P_{2}$, but the $P_{1}-P_{2}$ recursion is already included in the diagram (2.4), and introducing a third pencil would be redundant. In the recursion diagram, all structures appear on equal footing; on the other hand, in the applications that we have in mind there is a distinguished structure, so the "symmetry breaking" caused by the choice of two pencils is significant.

We stress that, given three Poisson structures $P_{0}, P_{1}$ and $P_{2}$, mutually compatible and such that the two pencils $P_{1}-\lambda P_{0}, P_{2}-\mu P_{0}$ both admit Casimir functions, a common Casimir function as required in Prop.(2.2) may not exist at all. An obvious necessary condition is that at each point $x$ of the phase space $\mathcal{M}$, and for generic values of the spectral parameters $(\lambda, \mu)$, the two subspaces $\operatorname{ker}\left(P_{1}-\lambda P_{0}\right)$ and $\operatorname{ker}\left(P_{2}-\mu P_{0}\right)$ should have a nontrivial intersection in $T_{x}^{*} \mathcal{M}$. For instance, let us consider the space $\mathbb{R}^{5}$, with coordinates $\left\{x_{1}, x_{2}, x_{3}, x_{4}, x_{5}\right\}$, endowed with the three Poisson tensors:

$$
\begin{aligned}
& P_{0}=\left(\begin{array}{ccccc}
0 & 0 & 1 & 0 & 0 \\
0 & 0 & 0 & 1 & 0 \\
-1 & 0 & 0 & 0 & 0 \\
0 & -1 & 0 & 0 & 0 \\
0 & 0 & 0 & 0 & 0
\end{array}\right) \\
& P_{1}=\left(\begin{array}{ccccc}
0 & 0 & 0 & 1 & 0 \\
0 & 0 & 0 & 0 & 1 \\
0 & 0 & 0 & 0 & 0 \\
-1 & 0 & 0 & 0 & 0 \\
0 & -1 & 0 & 0 & 0
\end{array}\right) \quad P_{2}=\left(\begin{array}{ccccc}
0 & 0 & 0 & 1 & 0 \\
0 & 0 & 0 & 0 & 0 \\
0 & 0 & 0 & 0 & 1 \\
-1 & 0 & 0 & 0 & 0 \\
0 & 0 & -1 & 0 & 0
\end{array}\right)
\end{aligned}
$$

Although the pencils $P_{1}-\lambda P_{0}$ and $P_{2}-\lambda P_{0}$ admit the Casimir functions $x_{3}+\lambda x_{4}+\lambda^{2} x_{5}$ and $x_{2}+\mu x_{1}-\mu^{2} x_{5}$, respectively, it is easy to check that a common Casimir function for both pencils does not exist.

The simple local geometry of our trihamiltonian structures may be clarified by an example. Let us consider the "fundamental molecule" (1.27). The lowest dimension in which this diagram can be realised is 9 . In fact, the diagram includes six functions, that we assume to be independent. Since the three vectorfields in the diagram commute, by Frobenius' theorem there exists a coordinate system in which they coincide with coordinate vectorfields: $X_{i} \equiv \frac{\partial}{\partial x^{i}}$ for $i=1,2,3$. The diagram shows that the hamiltonian $h_{0}^{0}$ is $P_{0}$-conjugate to $x^{1}$, while $h_{1}^{0}$ and $h_{0}^{1}$ are $P_{0}$-conjugate to $x^{2}$ and $x^{3}$ respectively. The other hamiltonians $h_{2}^{0}, h_{1}^{1}$ and $h_{0}^{2}$ are Casimir functions for $P_{0}$. Therefore, the 9 
functions $x^{i}$ and $h_{j}^{i}$ should be functionally independent, and locally form a coordinate system: let $x^{4} \equiv h_{0}^{0}, x^{5} \equiv h_{1}^{0}, x^{6} \equiv h_{0}^{1}, x^{7} \equiv h_{2}^{0}, x^{8} \equiv h_{1}^{1}$ and $x^{9} \equiv h_{0}^{2}$. It can be read directly from the diagram (1.27) that in these coordinates the three tensors $P_{0}, P_{1}$ and $P_{2}$ have the following matrix components:

$$
\begin{aligned}
& P_{0}=\left(\begin{array}{ccccccccc}
0 & 0 & 0 & 1 & 0 & 0 & 0 & 0 & 0 \\
0 & 0 & 0 & 0 & 1 & 0 & 0 & 0 & 0 \\
0 & 0 & 0 & 0 & 0 & 1 & 0 & 0 & 0 \\
-1 & 0 & 0 & 0 & 0 & 0 & 0 & 0 & 0 \\
0 & -1 & 0 & 0 & 0 & 0 & 0 & 0 & 0 \\
0 & 0 & -1 & 0 & 0 & 0 & 0 & 0 & 0 \\
0 & 0 & 0 & 0 & 0 & 0 & 0 & 0 & 0 \\
0 & 0 & 0 & 0 & 0 & 0 & 0 & 0 & 0 \\
0 & 0 & 0 & 0 & 0 & 0 & 0 & 0 & 0
\end{array}\right) \\
& P_{1}=\left(\begin{array}{ccccccccc}
0 & 0 & 0 & 0 & 1 & 0 & 0 & 0 & 0 \\
0 & 0 & 0 & 0 & 0 & 1 & 0 & 0 & 0 \\
0 & 0 & 0 & 0 & 0 & 0 & 1 & 0 & 0 \\
0 & 0 & 0 & 0 & 0 & 0 & 0 & 0 & 0 \\
-1 & 0 & 0 & 0 & 0 & 0 & 0 & 0 & 0 \\
0 & -1 & 0 & 0 & 0 & 0 & 0 & 0 & 0 \\
0 & 0 & -1 & 0 & 0 & 0 & 0 & 0 & 0 \\
0 & 0 & 0 & 0 & 0 & 0 & 0 & 0 & 0 \\
0 & 0 & 0 & 0 & 0 & 0 & 0 & 0 & 0
\end{array}\right) \quad P_{2}=\left(\begin{array}{ccccccccc}
0 & 0 & 0 & 0 & 0 & 1 & 0 & 0 & 0 \\
0 & 0 & 0 & 0 & 0 & 0 & 0 & 1 & 0 \\
0 & 0 & 0 & 0 & 0 & 0 & 0 & 0 & 1 \\
0 & 0 & 0 & 0 & 0 & 0 & 0 & 0 & 0 \\
0 & 0 & 0 & 0 & 0 & 0 & 0 & 0 & 0 \\
-1 & 0 & 0 & 0 & 0 & 0 & 0 & 0 & 0 \\
0 & 0 & 0 & 0 & 0 & 0 & 0 & 0 & 0 \\
0 & -1 & 0 & 0 & 0 & 0 & 0 & 0 & 0 \\
0 & 0 & -1 & 0 & 0 & 0 & 0 & 0 & 0
\end{array}\right)
\end{aligned}
$$

The fact that three independent Poisson tensor can be simultaneously put in canonical form is possible only because they are all degenerate (in the realization of minimal dimension, they ought to have the same rank), and their symplectic foliations are different. Having anticipated that the diagram (1.27) corresponds to the trihamiltonian structure of $\mathfrak{g l}(3)$, we have in fact shown that the latter trihamiltonian space admits local multicanonical coordinates. On the other hand, although six of the multicanonical coordinates coincide with the coefficients of the characteristic determinant of the Lax matrix $A \lambda+M$, the first three coordinates can be found only upon explicit integration of the dynamical system. Analogous considerations hold for the "fundamental molecule" of any space $\mathfrak{g l}(n)^{\kappa}$, and actually for any finite trihamiltonian recursion diagram (under the assumption that all the hamiltonians are independent, which is generically true for $\left.\mathfrak{g l}(n)^{\kappa}\right)$. The lowest dimension to accommodate a trihamiltonian structure admitting multicanonical coordinates is 4 (the "fundamental molecule" contains just one vectors field and three hamiltonians); an example is the algebra $\mathfrak{g l}(2)$. The reader can easily find out the multicanonical form of the three Poisson tensors generating the $\mathfrak{g l}(2)^{2}$ diagram (1.28).

Another type of coordinates, the separation coordinates, can instead be obtained explicitly in an alternative way, which does not require the integration of the vectorfields. This is explained in the next section.

\section{Separation of variables}

\subsection{Darboux-Nijenhuis coordinates}

In this section we shall adapt to the trihamiltonian framework the construction of separation variables proposed by Falqui, Magri and Pedroni in [0] [3]. 
The basic notion involved in their construction is the definition of Darboux-Nijenhuis coordinates [4] [7. Consider a bihamiltonian structure $P Q$ on a manifold $\mathcal{M}$, with $\operatorname{dim}(\mathcal{M})=2 m$, such that at least one of the Poisson tensors (say, $P$ ) is nondegenerate. Let $N$ be the recursion operator defined by (2.3); following Magri [4], we say that $\mathcal{M}$ is endowed with a Poisson-Nijenhuis structure. We shall assume that $P$ and $Q$ are such that at generic point of $\mathcal{M}$, the recursion tensor $N$ has $m$ distinct (double) eigenvalues $\lambda_{i}$. If moreover the $m$ eigenvalues $\lambda_{i}$ are functionally independent when regarded as functions on $\mathcal{M}$, then it has been proved [7] that (at least locally) other $m$ functions $\mu_{i}$ exist such that:

(i) the functions $\left(\lambda_{i}, \mu_{i}\right)$ form a system of coordinates on $\mathcal{M}$;

(ii) in this coordinate system, the Poisson tensor $P$ is in canonical form, i.e. $\left\{\lambda_{i}, \mu_{j}\right\}_{P}=$ $\delta_{i j}$ and $\left\{\lambda_{i}, \lambda_{j}\right\}_{P}=\left\{\mu_{i}, \mu_{j}\right\}_{P}=0$, and the recursion tensor $N$ is diagonal.

The coordinates $\left(\lambda_{i}, \mu_{i}\right)$ are called Darboux-Nijenhuis coordinates. The property (ii) completely determines the $Q$-Poisson brackets of the coordinates: $\left\{\lambda_{i}, \mu_{i}\right\}_{Q}=\lambda_{i}$ and $\left\{\lambda_{i}, \mu_{j}\right\}_{Q}=\left\{\lambda_{i}, \lambda_{j}\right\}_{Q}=\left\{\mu_{i}, \mu_{j}\right\}_{Q}=0$ for $i \neq j$.

Suppose $h_{i}$ to be a set of $m$ hamiltonians, independent and in involution with respect to $P$ and $Q$ (although not necessarily generated by Magri-Lenard recursion). Falqui, Magri and Pedroni [2] [3] have recently found an intrinsic coupling condition with the recursion operator, ensuring that all the functions $h_{i}$ are separable in the DarbouxNijenhuis coordinates.

In the case of a degenerate $P Q$ structure, one can sometimes perform a reduction onto a symplectic leaf of $P$, by projection along appropriate transversal vectorfields. This allows one to compute explicitly not only the coordinates $\lambda_{i}$ (which are the roots of the characteristic polynomial of $N$, or rather of its minimal polynomial), but also the other coordinates $\mu_{i}$, as the values taken by a suitable polynomial $p(\lambda)$ after the substitutions $\lambda=\lambda_{i}$ (for the construction of $p(\lambda)$, the exact statements and the proofs we refer the reader to [24]).

For a trihamiltonian structure $\left(P_{0}, P_{1}, P_{2}\right)$ with a nondegenerate Poisson tensor $P_{0}$, one is naturally led to introduce two recursion operators,

$$
N_{1}=P_{1} \cdot P_{0}^{-1} \quad \text { and } \quad N_{2}=P_{2} \cdot P_{0}^{-1},
$$

so obtaining a compatible pair of Poisson-Nijenhuis structures. In this case, under appropriate conditions it is possible to obtain trihamiltonian Darboux-Nijenhuis coordinates, having the most simple and natural property that one could imagine in this context:

Proposition 3.3: Let $N_{1}, N_{2}$ be the two tensors defined by (3.1) from a compatible trihamiltonian structure on a $2 m$-dimensional manifold. If

1. all the eigenspaces of $N_{1}$ and $N_{2}$ coincide (equivalently, $N_{1}$ and $N_{2}$ have the same centraliser);

2. both $N_{1}$ and $N_{2}$ have $m$ distinct eigenvalues, forming together a set of $2 m$ independent functions, that we denote by $\lambda_{i}$ and $\mu_{i}$ respectively $(i=1, \ldots, m)$; 
3. for any pair of eigenvalues $\lambda_{i}$ and $\mu_{i}$, corresponding to the same common eigenspace, one has

$$
\left\{\lambda_{i}, \mu_{i}\right\}_{P_{0}}=1
$$

then the eigenvalues of $N_{1}$ and $N_{2}$ (respectively denoted by $\lambda_{i}$ and $\mu_{i}$ ) form a DarbouxNijenhuis coordinate system.

Proof: For any Nijenhuis recursion operator $N$ and any of its eigenvaules $\lambda$, it is always true (see [4]) that $N^{*} d \lambda=\lambda d \lambda$. The fact that $N_{1}$ has $m$ independent eigenvalues implies that all its eigenspaces are bidimensional, and the same holds for $N_{2}$. We have also assumed that the eigenvalues of $N_{1}$ and $N_{2}$ corresponding to the $i$-th common eigenspace are independent functions. Therefore, the eigenspace itself is spanned by the two differentials $d \lambda_{i}$ and $d \mu_{i}$ :

$$
\begin{array}{ll}
N_{1}^{*} d \lambda_{i}=\lambda_{i} d \lambda_{i}, & N_{2}^{*} d \lambda_{i}=\mu_{i} d \lambda_{i}, \\
N_{1}^{*} d \mu_{i}=\lambda_{i} d \mu_{i}, & N_{2}^{*} d \mu_{i}=\mu_{i} d \mu_{i} .
\end{array}
$$

From the definition (3.1) and the trivial fact that $N_{1} P_{0}=P_{1}=P_{0} N_{1}^{*}$, at any point where $\lambda_{j} \neq 0$ one has

$$
\begin{aligned}
\left\{\lambda_{i}, \lambda_{j}\right\}_{P_{0}} & =\frac{1}{\lambda_{j}}\left\langle P_{0} d \lambda_{i}, \lambda_{j} d \lambda_{j}\right\rangle=\frac{1}{\lambda_{j}}\left\langle P_{0} d \lambda_{i}, N_{1}^{*} d \lambda_{j}\right\rangle \\
& =\frac{1}{\lambda_{j}}\left\langle P_{0} N_{1}^{*} d \lambda_{i}, d \lambda_{j}\right\rangle=\frac{\lambda_{i}}{\lambda_{j}}\left\{\lambda_{i}, \lambda_{j}\right\}_{P_{0}}
\end{aligned}
$$

and, since $\lambda_{i} \neq \lambda_{j}$ for $i \neq j$, one should have $\left\{\lambda_{i}, \lambda_{j}\right\}_{P_{0}}=0$ for all $i, j$. In a similar way, using the recursion tensor $N_{2}$ one obtains $\left\{\mu_{i}, \mu_{j}\right\}_{P_{0}}=0$ for all $i, j$. Furthermore,

$$
\begin{aligned}
\left\{\lambda_{i}, \mu_{j}\right\}_{P_{0}} & =\frac{1}{\lambda_{j}}\left\langle P_{0} d \lambda_{i}, \lambda_{j} d \mu_{j}\right\rangle=\frac{1}{\lambda_{j}}\left\langle P_{0} d \lambda_{i}, N_{1}^{*} d \mu_{j}\right\rangle \\
& =\frac{1}{\lambda_{j}}\left\langle P_{0} N_{1}^{*} d \lambda_{i}, d \mu_{j}\right\rangle=\frac{\lambda_{i}}{\lambda_{j}}\left\{\lambda_{i}, \mu_{j}\right\}_{P_{0}}
\end{aligned}
$$

which entails $\left\{\lambda_{i}, \mu_{j}\right\}_{P_{0}}=0$ for $i \neq j$. The above results extend by continuity to points where $\lambda_{j}=0$. The additional normalisation condition (3) ensures that $\left(\lambda_{i}, \mu_{i}\right)$ are canonical coordinates for $P_{0}$; by construction, both tensors $N_{1}$ and $N_{2}$ are diagonal in these coordinates, which therefore are Darboux-Nijenhuis for the trihamiltonian structure.

In other terms, if two Poisson pencils are available, and the two recursion operators are "independent" and "compatible" in the sense given above, then all the Darboux-Nijenhuis variables are obtained as eigenvalues (in the usual bihamiltonian setup, only half of these variables are defined as eigenvalues). The theorem also clarifies 
that trihamiltonian structures generated by single recursion operator, $P_{1}=N P_{0}$ and $P_{2}=N P_{1}=N^{2} P_{0}$, sometimes encountered in the literature, are not suitable for our purposes.

To obtain a twofold Poisson-Nijenhuis manifold also when the Poisson tensor $P_{0}$ is degenerate, we exploit a projection technique, which has been already used for a different purpose in [25].

Let us start with two compatible Poisson tensors $P_{0}$ and $P_{1}$. The rank of $P_{0}$ is assumed for simplicity to be constant on the phase manifold $\mathcal{M}$ (in the sequel, $\operatorname{dim} \mathcal{M}=2 m+k, k$ being the corank of $\left.P_{0}\right)$. The kernel of $P_{0}$ is then pointwise spanned by the differentials of $k$ Casimir functions $c^{\alpha}$. The main ingredient in our approach is a set of $k$ independent vectorfields $Z_{\alpha}$ (spanning a distribution $\mathcal{Z}$ ) having these properties:

$$
\begin{array}{rll}
\text { i) } & \text { normalization: } & Z_{\alpha}\left(c^{\beta}\right)=\delta_{\alpha}^{\beta} ; \\
\text { ii) } & \text { integrability: } & {\left[Z_{\alpha}, Z_{\beta}\right] \in \mathcal{Z} ;} \\
\text { iii) } & \text { symmetry for } P_{0}: & \mathcal{L}_{Z_{\alpha}} P_{0}=0 .
\end{array}
$$

The normalization property (i) entails that all the vectorfields $Z_{\alpha}$ are transversal to the symplectic leaves of $P_{0}$. The concrete possibility of finding such a set of transversal vectorfields is not ensured a priori. Here we shall assume their existence, but in the last section of the article we will give an explicit recipe to find them for a relevant class of trihamiltonian structures.

The vectorfields $Z_{\alpha}$ induce a decomposition on $T \mathcal{M}$ and $T^{*} \mathcal{M}$ :

$$
\begin{array}{ll}
\forall \theta \in T^{*} \mathcal{M} \quad\left\{\begin{array}{l}
\theta_{\perp}=\left\langle Z_{\alpha}, \theta\right\rangle \mathrm{d} c^{\alpha} \\
\theta_{/ /}=\theta-\theta_{\perp}
\end{array}\right. \\
\forall X \in T \mathcal{M} \quad\left\{\begin{array}{l}
X_{\perp}=X\left(c^{\alpha}\right) Z_{\alpha} \\
X_{/ /}=X-X_{\perp}
\end{array}\right.
\end{array}
$$

Lemma 3.4: The decomposition satisfies the following properties:

$$
\begin{aligned}
\left\langle\theta_{/ /}, Z_{\alpha}\right\rangle & =0, \\
X_{/ /}\left(c^{\alpha}\right) & =0 .
\end{aligned}
$$

Proof: $\left\langle\theta_{/ /}, Z_{\alpha}\right\rangle=\left\langle\theta-\theta_{\perp}, Z_{\alpha}\right\rangle=\left\langle\theta, Z_{\alpha}\right\rangle-\left\langle\theta, Z_{\beta}\right\rangle Z_{\alpha}\left(c^{\beta}\right)=0$; $X_{/ /}\left(c^{\alpha}\right)=X\left(c^{\alpha}\right)-X_{\perp}\left(c^{\alpha}\right)=X\left(c^{\alpha}\right)-X\left(c^{\beta}\right) Z_{\beta}\left(c^{\alpha}\right)=0$.

Using the above-defined decomposition, one proves that 
Lemma 3.5: The assumptions (3.2) imply $\left[Z_{\alpha}, Z_{\beta}\right]=0$.

Proof: $\left[Z_{\alpha}, Z_{\beta}\right] \in \mathcal{Z}$ entails $\left[Z_{\alpha}, Z_{\beta}\right]_{/ /}=0$, but one also has $\left[Z_{\alpha}, Z_{\beta}\right]_{\perp}=0$ because $\left[Z_{\alpha}, Z_{\beta}\right]\left(c^{\gamma}\right)=Z_{\alpha}\left(\delta_{\beta}^{\gamma}\right)-Z_{\beta}\left(\delta_{\alpha}^{\gamma}\right)=0$.

We can introduce a new tensor $\tilde{P}_{1}$ by setting

$$
\tilde{P}_{1}(\theta, \phi)=P_{1}\left(\theta_{/ /}, \phi / /\right)
$$

the kernel of the "deformed" tensor $\tilde{P}_{1}$ contains all the differentials of the Casimir functions of $P_{0}$. Still, the tensor $\tilde{P}_{1}$ is not automatically a Poisson tensor.

From the beginning, we have assumed that $\mathcal{L}_{Z_{\alpha}} P_{0}=0$ (3.2). This is equivalent to requiring that $P_{0}$ be projectable along the flows of the vectorfields $Z_{\alpha}$, i.e. for any pair of functions $(f, g)$ such that $Z_{\alpha}(f)=Z_{\alpha}(g)=0$ everywhere, their Poisson bracket should also be constant along the same flows: $Z_{\alpha}\left(\{f, g\}_{P_{0}}\right)=0$.

So far, nothing ensures that the new tensor $\tilde{P}_{1}$ is projectable in the same sense. Whenerver it turns out to be so, i.e.

$$
\mathcal{L}_{Z_{\alpha}} \tilde{P}_{1}=0
$$

then the $\tilde{P}_{1}$-bracket $\tilde{P}_{1}(d f, d g)$ can be reduced to $Z_{\alpha}$-invariant functions as well. For a $Z_{\alpha}$-invariant function $f$ one has $d f=(d f)_{/ /}$, so the $\tilde{P}_{1}$-bracket of such functions coincide with their $P_{1}$-bracket. Then, if $\tilde{P}_{1}$ is projectable, the Jacobi identity is straightfowardly proved for $Z_{\alpha}$-invariant functions. We claim that (3.4), supplemented by some auxiliary conditions, ensures both the Jacobi identity and the compatibility with $P_{0}$ for the $\tilde{P}_{1}^{-}$ bracket of arbitrary functions on $\mathcal{M}$.

Proposition 3.6: If (i) all the vectorfields $Z_{\alpha}$ fulfill both (3.2) and (3.4);

(ii) all the Casimir functions $c^{\alpha}$ are in mutual involution with respect to $P_{1}$, and

(iii) the functions $c^{\alpha}$ generate bihamiltonian vectorfields, i.e. one can find $k$ functions $h^{\alpha}$ such that $V^{\alpha} \equiv P_{1} \mathrm{~d} c^{\alpha}=P_{0} \mathrm{~d} h^{\alpha}$,

then $\tilde{P}_{1}$ is a Poisson tensor compatible with $P_{0}$.

The proof requires several steps.

Lemma 3.7: If the vectorfields $V^{\alpha} \equiv P_{1} \mathrm{~d} c^{\alpha}$ are hamiltonian also with respect to $P_{0}$, then the Poisson tensor $P_{1}$ and the tensor $\tilde{P}_{1}$ differ by a Lie derivative of $P_{0}$ :

$$
\tilde{P}_{1}=P_{1}+\mathcal{L}_{X_{P_{1}}} P_{0},
$$

where (summation is understood on repeated indices)

$$
X_{P_{1}}=h^{\alpha} Z_{\alpha}
$$


Proof: One has

$$
\begin{aligned}
\tilde{P}_{1}(d f, d g)= & P_{1}\left((d f)_{/ /},(d g)_{/ /}\right)=P_{1}\left(d f-(d f)_{\perp}, d g-(d g)_{\perp}\right) \\
= & P_{1}(d f, d g)+Z_{\alpha}(f) Z_{\beta}(g) P_{1}\left(d c^{\alpha}, d c^{\beta}\right)- \\
& -Z_{\alpha}(g) Z_{\beta}(g) P_{1}\left(d f, d c^{\alpha}\right)-Z_{\alpha}(f) P_{1}\left(d c^{\alpha}, d g\right) \\
= & P_{1}(d f, d g)-Z_{\alpha}(g) Z_{\beta}(g) P_{0}\left(d f, d h^{\alpha}\right)-Z_{\alpha}(f) P_{0}\left(d h^{\alpha}, d g\right)
\end{aligned}
$$

which proves the statement since

$$
\begin{aligned}
\left(\mathcal{L}_{X_{P_{1}}} P_{0}\right)(d f, d g)= & h^{\alpha}\left[Z_{\alpha}\left(\{f, g\}_{P_{0}}\right)-\left\{Z_{\alpha}(f), g\right\}_{P_{0}}-\left\{f, Z_{\alpha}(g)\right\}_{P_{0}}\right]- \\
& -Z_{\alpha}(f)\left\{h^{\alpha}, g\right\}_{P_{0}}-Z_{\alpha}(g)\left\{f, h^{\alpha}\right\}_{P_{0}} \\
= & h^{\alpha}\left[\left(\mathcal{L}_{Z_{\alpha}} P_{0}\right)(d f, d g)\right]-Z_{\alpha}(f)\left\{h^{\alpha}, g\right\}_{P_{0}}-Z_{\alpha}(g)\left\{f, h^{\alpha}\right\}_{P_{0}} \\
= & -Z_{\alpha}(f)\left\{h^{\alpha}, g\right\}_{P_{0}}-Z_{\alpha}(g)\left\{f, h^{\alpha}\right\}_{P_{0}} .
\end{aligned}
$$

This also proves that, under the given hypotheses, $\mathcal{L}_{X_{P_{1}}} P_{0}=V^{\alpha} \wedge Z_{\alpha}$.

If condition (iii) in the statement of Prop.(3.6) is fulfilled, then (3.4) can be rewritten in terms of the tensor $P_{1}$, using the previous Lemma:

Lemma 3.8: Upon assuming (3.5), 3.4) is equivalent to

$$
\mathcal{L}_{Z_{\alpha}} P_{1}=\left[V^{\beta}, Z_{\alpha}\right] \wedge Z_{\beta}
$$

Proof: Here and in the sequel we exploit the properties of the Schouten bracket, a bilinear operator on contravariant, antisymmetric tensors of arbitrary rank $p$ ( $p$-vectors). We refer the reader to [Vaisman] for the general theory; the properties that we shall use are the following:

- if $P$ is a $p$-vector and $Q$ is a $q$-vector, then $[P, Q]_{\mathrm{S}}=(-1)^{p q}[Q, P]_{\mathrm{S}}$;

- if, moreover, $R$ is a $r$-vector, $[P, Q \wedge R]_{\mathrm{S}}=[P, Q]_{\mathrm{S}} \wedge R+(-1)^{q(p+1)} Q \wedge[P, R]_{\mathrm{S}}$;

- if $X$ is an ordinary vectorfield, then $[X, P]_{\mathrm{S}} \equiv \mathcal{L}_{X} P$

- $\mathcal{L}_{X}[P, Q]_{\mathrm{S}}=\left[\mathcal{L}_{X} P, Q\right]_{\mathrm{S}}+\left[P, \mathcal{L}_{X} Q\right]_{\mathrm{S}} ;$

- if $P$ is a bivector, then it is a Poisson tensor if and only if $[P, P]_{\mathrm{S}}=0$;

- if $P$ and $Q$ are both Poisson tensors, then they are compatible if and only if $[P, Q]_{\mathrm{S}}=0$. 
Proving the Lemma is now straightforward:

$$
\begin{aligned}
\mathcal{L}_{Z_{\alpha}} P_{1} & =\mathcal{L}_{Z_{\alpha}}\left(\tilde{P}_{1}-\mathcal{L}_{X_{P_{1}}} P_{0}\right) \\
& =-\mathcal{L}_{Z_{\alpha}} \mathcal{L}_{X_{P_{1}}} P_{0} \\
& =-\left[Z_{\alpha}, V^{\beta} \wedge Z_{\beta}\right]_{\mathrm{S}} \\
& =-\left[Z_{\alpha}, V^{\beta}\right] \wedge Z_{\beta}-V^{\beta} \wedge\left[Z_{\alpha}, Z_{\beta}\right] \\
& =\left[V^{\beta}, Z_{\alpha}\right] \wedge Z_{\beta}
\end{aligned}
$$

The condition (3.7) is also a projectability condition: it means that the tensor $P_{1}$ itself can be reduced to $Z_{\alpha}$-invariant functions, but in this case one cannot identify the image of $P_{1}$ through projection (on the quotient space) with the restriction of $P_{1}$ on a submanifold transversal to the vectorfields $Z_{\alpha}$ (for instance, a symplectic leaf of $P_{0}$ ). As a matter of fact, we have seen that the projection of $P_{1}$ would instead coincide with the restriction of $\tilde{P}_{1}$.

Now we can eventually prove the main statement.

Proof: We need to show that $\left[\tilde{P}_{1}, \tilde{P}_{1}\right]_{\mathrm{S}}=0$ and $\left[P_{0}, \tilde{P}_{1}\right]_{\mathrm{S}}=0$. The second equality is easy to prove. By assumption, $\left[P_{0}, P_{0}\right]_{\mathrm{S}}=\left[P_{1}, P_{1}\right]_{\mathrm{S}}=\left[P_{0}, P_{1}\right]_{\mathrm{S}}=0$. Taking the Lie derivative of $\left[P_{0}, P_{0}\right]_{\mathrm{S}}$ one finds that $\left[P_{0}, \mathcal{L}_{X} P_{0}\right]_{\mathrm{S}}=0$ for any vectorfield $X$, and from (3.5) it follows that $\left[P_{0}, \tilde{P}_{1}\right]_{\mathrm{S}}=\left[P_{0}, P_{1}+\mathcal{L}_{X_{P_{1}}} P_{0}\right]_{\mathrm{S}} \equiv 0$. To prove the first equality, observe furthemore that the vectorfields $V^{\alpha}$ are assumed to be hamiltonian for $P_{1}$, hence $\left[P_{1}, V^{\alpha}\right]_{\mathrm{S}} \equiv \mathcal{L}_{V^{\alpha}} P_{1}=0$. Then

$$
\begin{aligned}
{\left[\tilde{P}_{1}, \tilde{P}_{1}\right]_{\mathrm{S}}=} & {\left[P_{1}, P_{1}\right]_{\mathrm{S}}+2\left[P_{1}, V^{\alpha} \wedge Z_{\alpha}\right]_{\mathrm{S}}+\left[\mathcal{L}_{X_{P_{1}}} P_{0}, V^{\alpha} \wedge Z_{\alpha}\right]_{\mathrm{S}} } \\
= & 2\left[P_{1}, V^{\alpha}\right]_{\mathrm{S}} \wedge Z_{\alpha}-2 V^{\alpha} \wedge\left[P_{1}, Z_{\alpha}\right]_{\mathrm{S}}+ \\
& {\left[\mathcal{L}_{X_{P_{1}}} P_{0}, V^{\alpha}\right]_{\mathrm{S}} \wedge Z_{\alpha}-V^{\alpha} \wedge\left[\mathcal{L}_{X_{P_{1}}} P_{0}, Z_{\alpha}\right]_{\mathrm{S}} } \\
= & 2 V^{\alpha} \wedge\left[Z_{\alpha}, V^{\beta}\right] \wedge Z_{\beta}+\left[V^{\alpha}, V^{\beta} \wedge Z_{\beta}\right]_{\mathrm{S}} \wedge Z_{\alpha}-V^{\alpha} \wedge\left[Z_{\alpha}, V^{\beta} \wedge Z_{\beta}\right]_{\mathrm{S}} \\
= & 2 V^{\alpha} \wedge\left[Z_{\alpha}, V^{\beta}\right] \wedge Z_{\beta}+\left[V^{\alpha}, V^{\beta}\right] \wedge Z_{\beta} \wedge Z_{\alpha}+V^{\beta} \wedge\left[V^{\alpha}, Z_{\beta}\right] \wedge Z_{\alpha}- \\
& V^{\alpha} \wedge\left[Z_{\alpha}, V^{\beta}\right] \wedge Z_{\beta}-V^{\alpha} \wedge V^{\beta} \wedge\left[Z_{\alpha}, Z_{\beta}\right] \\
= & 2 V^{\alpha} \wedge\left[Z_{\alpha}, V^{\beta}\right] \wedge Z_{\beta}-V^{\alpha} \wedge\left[Z_{\alpha}, V^{\beta}\right] \wedge Z_{\beta}-V^{\alpha} \wedge\left[Z_{\alpha}, V^{\beta}\right] \wedge Z_{\beta} \\
= & 0
\end{aligned}
$$

We have seen that $P_{0}$ and $\tilde{P}_{1}$ are compatible and can both be reduced by projection along the flows of the vectorfields $Z_{\alpha}$ on any symplectic leaf of $P_{0}$. Then, on each leaf one can define a recursion tensor and look for Darboux-Nijenhuis coordinates; but there is no evidence that such coordinates can be extended to some neighborhood of the 
symplectic leaf in $\mathcal{M}$. However, a Nijenhuis tensor $N_{1}$ can be directly defined on the full manifold $\mathcal{M}$ in the following way. To any vectorfield $X$ over $\mathcal{M}$ one can associate the vectorfield $X_{/ /}$which is, by construction, everywhere tangent to the simplectic leaves of $P_{0}$. Consider now any one-form $\theta_{X}$ such that $X_{/ /}=P_{0} \theta_{X}$; we set

$$
N_{1} X=\tilde{P}_{1} \theta_{X}
$$

The tensor $N_{1}$ is actually independent of the choice of $\theta_{X}$, because the latter is defined up to an element of the kernel of $P_{0}$, which is also the kernel of $\tilde{P}_{1}$. From the definition, it follows immediately that $N_{1} Z_{\alpha}=0$, and that for any hamiltonian vectorfield $X_{f}=P_{0} d f$ one has $N_{1} X_{f}=\tilde{P}_{1} d f$.

Proposition 3.9: Under the same hypotheses of the previous proposition, the tensor $N_{1}$ defined by (3.8) is a Nijenhuis tensor.

Proof: We need to show that the Nijenhuis torsion tensor $T_{N}$ of $N_{1}$ vanishes:

$$
T_{N}(X, Y) \equiv\left[N_{1} X, N_{1} Y\right]-N_{1}\left[N_{1} X, Y\right]-N_{1}\left[X, N_{1} Y\right]+N_{1}^{2}[X, Y]=0
$$

The Nijenhuis torsion tensor acts pointwise on vectors; thus, it is sufficient to show that $T_{N}(X, Y)$ vanishes on each pair of elements of a basis of the tangent space to $\mathcal{M}$ at any point. In our case, such a basis is provided by a set of $P_{0}$-hamiltonian vectorfields spanning the tangent spaces to the symplectic leaves, and by the transversal vectorfields $Z_{\alpha}$. We already know that $N_{1} Z_{\alpha}=0$ and $\left[Z_{\alpha}, Z_{\beta}\right]=0$, thus $T_{N}\left(Z_{\alpha}, Z_{\beta}\right)=0$ for any pair $(\alpha, \beta)$. More generally, for any vectorfield $X$

$$
\begin{aligned}
T_{N}\left(X, Z_{\alpha}\right) & =N_{1}\left(N_{1}\left[X, Z_{\alpha}\right]-\left[N_{1} X, Z_{\alpha}\right]\right) \\
& =N_{1}\left(\mathcal{L}_{Z_{\alpha}}\left(N_{1} X\right)-N_{1} \mathcal{L}_{Z_{\alpha}} X\right) \\
& =N_{1}\left(\mathcal{L}_{Z_{\alpha}}\left(N_{1}\right) P_{0} \theta_{X}\right)
\end{aligned}
$$

but since $\mathcal{L}_{Z_{\alpha}} \tilde{P}_{1}=\mathcal{L}_{Z_{\alpha}} P_{0}=0$, one has $\mathcal{L}_{Z_{\alpha}}\left(N_{1}\right) P_{0}=0$.

Finally, we evaluate the torsion tensor on two hamiltonian vectorfields $X_{f}=P_{0} d f$ and $X_{g}=P_{0} d g$; for notational convenience, we set $Y_{f}=P_{1} d f$. This part of the proof is well known and can be found, in greater detail, in [Marsico]: it is shown there that the compatibility of $\tilde{P}_{1}$ and $P_{0}$, i.e. $\left[P_{0}, \tilde{P}_{1}\right]_{\mathrm{S}}=0$, implies

$$
\left[X_{f}, Y_{g}\right]+\left[Y_{f}, X_{g}\right]=X_{\{f, g\}_{\tilde{P} 1}}+Y_{\{f, g\}_{P_{0}}} .
$$

Using the fact that $Y_{f}=N_{1} X_{f}$, applying once again $N_{1}$ to both sides of (3.9), and rearranging terms, one gets

$$
Y_{\{f, g\}_{\tilde{P}_{1}}}=N_{1}\left[X_{f}, N_{1} X_{g}\right]+N_{1}\left[N_{1} X_{f}, X_{g}\right]-N_{1}^{2}\left[X_{f}, X_{g}\right]
$$


on the oter hand, $Y_{\{f, g\}_{\tilde{P_{1}}}}=\left[Y_{f}, Y_{g}\right]=\left[N_{1} X_{f}, N_{1} X_{g}\right]$ and so

$$
T_{N}\left(X_{f}, X_{g}\right)=0
$$

These results suggest the following strategy: given a trihamiltonian structure, choose one of the Poisson tensors, say $P_{0}$; a complete set of Casimir functions $c^{\alpha}$ can be directly read out from the "fundamental molecule", as well as the functions $h^{\alpha}$ and $k^{\alpha}$ used in (3.6). In this case the vectorfields $P_{1} d c^{\alpha}$ and $P_{2} d c^{\alpha}$ are automatically commuting, so the conditions (ii) and (iii) of Prop.(3.6) are fulfilled. If one is able to find a complete set of vectorfields fulfilling all of $(3.2)$ and being symmetries of both $\tilde{P}_{1}$ and $\tilde{P}_{2}$ (in the sequel, we shortly write "a good set of transversal symmetries"), then one can apply the above procedure to each of the pairs $\left(P_{0}, P_{1}\right)$ and $\left(P_{0}, P_{2}\right)$ separately, obtaining in this way a pair of Nijenhuis tensors $N_{1}$ and $N_{2}$ on the full manifold $\mathcal{M}$.

This fact, however, does not yet ensure the existence of Darboux-Nijenhuis coordinates. The proof of the existence of Darboux-Nijenhuis coordinates has been given in Prop.(3.3) only for pairs of Nijenhuis tensors fulfilling some additional requirements, first of all that of being non-degenerate. We have not addressed the general problem of the existence of a canonical form for a non-regular Nijenhuis tensor; incidentally, to our knowledge non-regular Nijenhuis tensors have never been previously considered in connection with finite-dimensionl integrable systems. In the next section, we will rather characterise a particular class of trihamiltonian systems for which Darboux-Nijenhuis coordinates exist, are separation variables for the Hamiltonians occurring in the "fundamental molecule", and can even be constructed without having to compute eigenvalues. Although the assumptions that we make could seem rather artificial and difficult to test in practice, we will eventually show that this class of systems is not empty, and contains relevant examples described by Lax equations (with spectral parameter).

\subsection{Sklyanin separation of trihamiltonian systems}

We first recall the separability criterion introduced by Sklyanin 12. Let $h_{i}$ be $m$ hamiltonian in involution for a nondegenerate Poisson tensor $P$, and let $\left(\lambda_{i}, \mu_{i}\right)_{i=1, \ldots, m}$ a system of canonical coordinates for $P$. If $m$ functions $W_{i}$ of $m+2$ variables exist such that

$$
\begin{array}{cc}
W_{1}\left(\lambda_{1}, \mu_{1} ; h_{1}\left(\lambda_{i}, \mu_{i}\right), \ldots, h_{m}\left(\lambda_{i}, \mu_{i}\right)\right) & =0 \\
\vdots & \\
W_{m}\left(\lambda_{m}, \mu_{m} ; h_{1}\left(\lambda_{i}, \mu_{i}\right), \ldots, h_{m}\left(\lambda_{i}, \mu_{i}\right)\right) & =0
\end{array}
$$

identically, then all hamiltonians $h_{i}$ are separable in the coordinates $\left(\lambda_{i}, \mu_{i}\right)$. 
This setting refers to the symplectic case (as it should be, since the notion of separability of the Hamilton-Jacobi equation makes sense only in symplectic manifolds, namely on cotangent bundles). In the case of degenerate Poisson manifolds, one can discuss separability on symplectic leaves. Upon reduction, one may expect to find Sklyanin functions $W_{i}$ depending on auxiliary parameters labeling the symplectic leaves (in our case, the Casimir functions $c^{\alpha}$ ). This is indeed what happens in the example that we shall now discuss: first, we introduce a set of equations built using the common Casimir function $f_{\lambda \mu}$ (generating the "fundamental molecule") and the transversal vectorfields $Z_{\alpha}$, and we list sufficient conditions for the roots of these equations to form, together with the Casimir functions $c^{\alpha}$ ), a system of generalised Darboux-Nijenhuis coordinates $\left(\lambda_{i}, \mu_{i}, c^{\alpha}\right)$ on $\mathcal{M}$. Then, we produce $m$ functions $W_{i}$, each depending on the $i$-th pair of coordinates $\left(\lambda_{i}, \mu_{i}\right)$, on all the $k$ Casimir functions $c^{\alpha}$, and on other $m$ arguments. Once the latter are replaced by the remaining $m$ Hamiltonians $h_{b}^{a}\left(\lambda_{j}, \mu_{j}, c^{\alpha}\right)$ of the fundamental molecule, the functions $W_{i}$ vanish identically for any value of the coordinates $c^{\alpha}$, thus on all symplectic leaves simultaneously.

As previously, we assume that all the three Poison tensors $\left(P_{0}, P_{1}, P_{2}\right)$ have the same rank $2 m$, and the dimension of the manifold is $2 m+k$. We also assume that the common Casimir function $f_{\lambda \mu}$ is complete, i.e. that among its coefficients one can find $m+k$ independent functions, including $k$ Casimir functions for each of the three Poisson tensors (different tensors may indeed have some common Casimir functions). In the cases that we shall consider (for instance, the trihamiltonian spaces $\mathfrak{g l}(r)^{n}$ ), the polynomial $f_{\lambda \mu}$ has exactly $m+k$ non-constant coefficients.

The leading role in our construction is played by the derivatives of the common Casimir function $f_{\lambda \mu}$ along the transversal vectorfields $Z_{\alpha}$. We denote these $k$ functions (still depending on the two parameters $\lambda, \mu$ ) by

$$
S_{\alpha}(\lambda, \mu)=Z_{\alpha}\left(f_{\lambda \mu}\right)
$$

The letter $S$ is chosen because of the coincidence with Sklyanin's minors [12], in the particular case discussed in section (4).

Having assumed that the common Casimir function $f_{\lambda \mu}$ is polynomial in both parameters, the functions $S_{\alpha}(\lambda, \mu)$ are polynomials as well. We shall prove that, whenever appropriate conditions are verified, the common roots $\left(\lambda_{i}, \mu_{i}\right)$ of the polynomials $S_{\alpha}(\lambda, \mu)$ are Darboux-Nijenhuis coordinates and fulfill Sklyanin's separability condition (3.11). Note that we use the abbreviated notation $\left.F(\lambda \mu)\right|_{\lambda_{i}, \mu_{i}}$ for $\left.F(\lambda \mu)\right|_{\lambda=\lambda_{i}, \mu=\mu_{i}}$.

Proposition 3.10: If a good set of transversal symmetries $Z_{\alpha}$ fulfills in addition the following requirements:

1. all second directional derivatives of the complete common Casimir function $f_{\lambda \mu}$ vanish identically, i.e.

$$
Z_{\alpha}\left(Z_{\beta}\left(f_{\lambda \mu}\right)\right)=0 \quad \text { for all } \alpha, \beta
$$


2. the polynomials $S_{\alpha}(\lambda, \mu)=Z_{\alpha}\left(f_{\lambda \mu}\right)$ have $2 m$ functionally independent common roots $\left\{\lambda_{i}, \mu_{i}\right\}$;

3. the following equality holds, and for any $i$ both sides are not identically vanishing for at least one pair $(\alpha, \beta)$ :

$$
\left.\left\{S_{\alpha}(\lambda, \mu), S_{\beta}(\lambda, \mu)\right\}_{P_{0}}\right|_{\lambda_{i}, \mu_{i}}=\left.\left(\frac{\partial S_{\alpha}}{\partial \lambda} \frac{\partial S_{\beta}}{\partial \mu}-\frac{\partial S_{\alpha}}{\partial \mu} \frac{\partial S_{\beta}}{\partial \lambda}\right)\right|_{\lambda_{i}, \mu_{i}} ;
$$

then, the $2 m$ functions $\left(\lambda_{i}, \mu_{i}\right)$ form a system of Darboux-Nijenhuis coordinates on each symplectic leaf of $P_{0}$, and moreover $Z_{\alpha}\left(\lambda_{i}\right)=Z_{\alpha}\left(\mu_{i}\right)=0$.

Proof: First, we compute the parameter-dependent vectorfield associated to the common Casimir function $f_{\lambda \mu}$ under the deformed Poisson pencil $\left(\tilde{P}_{1}-\lambda P_{0}\right)$ :

$$
\left(\tilde{P}_{1}-\lambda P_{0}\right) d f_{\lambda \mu}=\left(P_{1}+\mathcal{L}_{X_{P_{1}}} P_{0}-\lambda P_{0}\right) d f_{\lambda \mu}=\left(\mathcal{L}_{X_{P_{1}}} P_{0}\right) d f_{\lambda \mu}
$$

But

$$
\left(\mathcal{L}_{X_{P_{1}}} P_{0}\right) d f_{\lambda \mu}=-\sum_{\alpha=1}^{k} S_{\alpha}(\lambda, \mu) \cdot P_{0} d h^{\alpha}:
$$

in fact, from the definition of the deformation vectorfields (3.6), one sees that the vectorfield $\left(\mathcal{L}_{X_{P_{1}}} P_{0}\right) d f_{\lambda \mu}$ acts on an arbitrary function $g$ as follows:

$$
\begin{aligned}
{\left[\left(\mathcal{L}_{X_{P_{1}}} P_{0}\right) d f_{\lambda \mu}\right](g) } & =-\sum_{\alpha=1}^{k} Z_{\alpha}\left(f_{\lambda \mu}\right)\left\{h^{\alpha}, g\right\}_{P_{0}}-\sum_{\alpha=1}^{k} Z_{\alpha}(g)\left\{f_{\lambda \mu}, h^{\alpha}\right\}_{P_{0}} \\
& =-\sum_{\alpha=1}^{k} S_{\alpha}(\lambda, \mu) \cdot\left(P_{0} d h^{\alpha}\right)(g) .
\end{aligned}
$$

Taking the derivative along $Z_{\alpha}$ of (3.14), one obtains

$$
\left(\tilde{P}_{1}-\lambda P_{0}\right) d S_{\alpha}(\lambda, \mu)=-\sum_{\beta=1}^{k} S_{\beta}(\lambda, \mu) \cdot P_{0} d\left[Z_{\alpha}\left(h^{\beta}\right)\right]
$$

(we used the fact that $Z_{\alpha}$ is a symmetry for both $P_{0}$ and $\tilde{P}_{1}$ ).

Since $\left(\lambda_{i}, \mu_{i}\right)$ is a pair of roots of $S_{\alpha}$, one finds that $\left.\left[\left(\tilde{P}_{1}-\lambda P_{0}\right) d S_{\alpha}(\lambda, \mu)\right]\right|_{\lambda_{i}, \mu_{i}}=0$ and that

$$
\begin{aligned}
0=d\left[S_{\alpha}\left(\lambda_{i}, \mu_{i}\right)\right]= & {\left.\left[d S_{\alpha}(\lambda, \mu)\right]\right|_{\lambda_{i}, \mu_{i}} } \\
& +\left.\frac{\partial S_{\alpha}}{\partial \lambda}\right|_{\lambda_{i}, \mu_{i}} d \lambda_{i}+\left.\frac{\partial S_{\alpha}}{\partial \mu}\right|_{\lambda_{i}, \mu_{i}} d \mu_{i} ;
\end{aligned}
$$


then,

$$
\left.\frac{\partial S_{\alpha}}{\partial \lambda}\right|_{\lambda_{i}, \mu_{i}}\left(\tilde{P}_{1} d \lambda_{i}-\lambda_{i} P_{0} d \lambda_{i}\right)+\left.\frac{\partial S_{\alpha}}{\partial \mu}\right|_{\lambda_{i}, \mu_{i}}\left(\tilde{P}_{1} d \mu_{i}-\lambda_{i} P_{0} d \mu_{i}\right)=0
$$

On the other hand,

$$
\left.Z_{\beta}\left(\lambda_{i}\right) \frac{\partial S_{\alpha}}{\partial \lambda}\right|_{\lambda_{i}, \mu_{i}}+\left.Z_{\beta}\left(\mu_{i}\right) \frac{\partial S_{\alpha}}{\partial \mu}\right|_{\lambda_{i}, \mu_{i}}=Z_{\beta}\left[S_{\alpha}\left(\lambda_{i}, \mu_{i}\right)\right]-\left.Z_{\beta}\left[S_{\alpha}(\lambda, \mu)\right]\right|_{\lambda_{i}, \mu_{i}}=0,
$$

having assumed $Z_{\beta}\left[S_{\alpha}(\lambda, \mu)\right]=0$ and $S_{\alpha}\left(\lambda_{i}, \mu_{i}\right)=0$. Let $\mathbf{S}$ be the $k \times 2$ matrix whose rows are $\left(\frac{\partial S_{\alpha}}{\partial \lambda}, \frac{\partial S_{\alpha}}{\partial \mu}\right)$, and let $\mathbf{S}_{(i)}$ denote the same matrix after the substitution $(\lambda, \mu) \rightarrow$ $\left(\lambda_{i}, \mu_{i}\right)$. The condition (3.13) entails that $\mathbf{S}_{(i)}$ has maximal rank for all $i=1, \ldots, m$ so the previous results imply that

$$
\begin{aligned}
Z_{\alpha}\left(\lambda_{i}\right) & =Z_{\alpha}\left(\mu_{i}\right)=0 \\
\tilde{P}_{1} d \lambda_{i} & =\lambda_{i} P_{0} d \lambda_{i} \\
\tilde{P}_{1} d \mu_{i} & =\lambda_{i} P_{0} d \mu_{i},
\end{aligned}
$$

and the same holds for $\tilde{P}_{2}$. Repeating now the same argument used in the proof of Prop.(3.3) one obtains that $\left\{\lambda_{i}, \lambda_{j}\right\}_{P_{0}}=0$ and $\left\{\mu_{i}, \mu_{j}\right\}_{P_{0}}=0$ for all $(i, j)$, and that $\left\{\lambda_{i}, \mu_{j}\right\}_{P_{0}}=0$ for $i \neq j$.

To get the remaining canonical bracket, one should instead rely on (3.13): for any pair of polynomials $F(\lambda, \mu)$ and $G(\lambda, \mu)$ such that $F\left(\lambda_{i}, \mu_{i}\right)=G\left(\lambda_{i}, \mu_{i}\right)=0$, one has

$$
\left.\{F(\lambda, \mu), G(\lambda, \mu)\}_{P_{0}}\right|_{\lambda_{i}, \mu_{i}}=\left.\left\{\lambda_{i}, \mu_{i}\right\}_{P_{0}}\left(\frac{\partial F}{\partial \lambda} \frac{\partial G}{\partial \mu}-\frac{\partial F}{\partial \mu} \frac{\partial G}{\partial \lambda}\right)\right|_{\lambda_{i}, \mu_{i}} ;
$$

then, if (3.13) holds and both sides are nonvanishing, one concludes

$$
\left\{\lambda_{i}, \mu_{i}\right\}_{P_{0}}=1 .
$$

Next, we prove Sklyanin's separability condition for the variables constructed according to the previous proposition.

Proposition 3.11: Let $\left(\lambda_{i}, \mu_{i}, c^{\alpha}\right)_{i=1, \ldots, m, \alpha=1, \ldots, k}$ be the generalised Darboux-Nijenhuis coordinates associated to the projection along the vectorfields $Z_{\alpha}$ according to Prop.(3.10). For each $i=1, \ldots, m$

$$
\left.f_{\lambda \mu}\right|_{\lambda_{i}, \mu_{i}}=p_{i}\left(\lambda_{i}, \mu_{i}\right)
$$

where $p_{i}(\lambda, \mu)$ are polynomials with constant coefficients. Hence, the coordinates $\left(\lambda_{i}, \mu_{i}\right)$ and the remaining $m$ hamiltonians $h_{b}^{a}$ (restricted to the symplectic leaf $\Sigma$ ) fulfill the separability condition (3.11), with

$$
W_{i}\left(\lambda_{i}, \mu_{i} ; h_{b}^{a}\right)=\left.f_{\lambda \mu}\right|_{\lambda_{i}, \mu_{i}}-p_{i}\left(\lambda_{i}, \mu_{i}\right)
$$


Proof: From the previous proposition, we know that

$$
S_{\alpha}\left(\lambda_{i}, \mu_{i}\right)=0, \quad Z_{\alpha}\left(\lambda_{i}\right)=0, \quad Z_{\alpha}\left(\mu_{i}\right)=0:
$$

together with the normalization condition (3.2. i), these imply that in the coordinate system $\left(\lambda_{i}, \mu_{i}, c^{\alpha}\right)$ one has

$$
Z_{a} \equiv \frac{\partial}{\partial c^{\alpha}}
$$

We denote the function obtained by replacing the spectral parameters $(\lambda, \mu)$ with the pair of coordinates $\left(\lambda_{i}, \mu_{i}\right)$ by $\left.f_{(i)} \equiv f_{\lambda_{\mu}}\right|_{\lambda_{i}, \mu_{i}}$. On account of (3.17),

$$
Z_{\alpha}\left(f_{(i)}\right)=S_{\alpha}\left(\lambda_{i}, \mu_{i}\right)=0
$$

thus the $m$ functions $f_{(i)}$, which would in principle depend on all the coordinates $\left(\lambda_{j}, \mu_{j}, c^{\alpha}\right)$, actually do not depend on the Casimir coordinates $c^{\alpha}$. To prove that they depend only on the pair $\left(\lambda_{i}, \mu_{i}\right)$, we exploit the properties of Darboux-Nijenhuis coordinates: for any function $g$, one has

$$
\left\{g, \lambda_{j}\right\}_{P_{0}}=\frac{\partial g}{\partial \mu_{j}} \quad \text { and } \quad\left\{g, \lambda_{j}\right\}_{\tilde{P}_{1}}=\lambda_{j} \frac{\partial g}{\partial \mu_{j}} .
$$

Thus, if for some $i$ one finds a function $g$ such that $\left\{g, \lambda_{j}\right\}_{\tilde{P}_{1}}-\lambda_{i}\left\{g, \lambda_{j}\right\}_{P_{0}}=0$ identically for any $j$, then $\left(\lambda_{j}-\lambda_{i}\right) \frac{\partial g}{\partial \mu_{j}} \equiv 0$ and the function $g$ cannot depend on $\mu_{j}$ for $j \neq i$. If furthermore $\left\{g, \mu_{j}\right\}_{\tilde{P}_{2}}-\mu_{i}\left\{g, \mu_{j}\right\}_{P_{0}}$ vanishes for any $j$ as well, then $g$ depends only on the pair $\left(\lambda_{i}, \mu_{i}\right)$. So what we need to prove is that, for any $j \neq i$,

$$
\left\{f_{(i)}, \lambda_{j}\right\}_{\tilde{P}_{1}}-\lambda_{i}\left\{f_{(i)}, \lambda_{j}\right\}_{P_{0}}=\left\{f_{(i)}, \mu_{j}\right\}_{\tilde{P}_{2}}-\mu_{i}\left\{f_{(i)}, \mu_{j}\right\}_{P_{0}}=0 .
$$

The differential of the function $f_{(i)}$ is given by

$$
d f_{(i)}=\left.\left(d f_{\lambda \mu}\right)\right|_{\lambda_{i}, \mu_{i}}+\left.\frac{\partial f_{\lambda \mu}}{\partial \lambda}\right|_{\lambda_{i}, \mu_{i}} d \lambda_{i}+\left.\frac{\partial f_{\lambda \mu}}{\partial \mu}\right|_{\lambda_{i}, \mu_{i}} d \mu_{i} .
$$

Thus, the vectorfield defined by applying the tensor $\left(\tilde{P}_{1}-\lambda_{i} P_{0}\right)$ to the differential of the function $f_{(i)}$ can be obtained from (3.14) by replacing the parameters $(\lambda, \mu)$ with the $i$-th pair of coordinates $\left(\lambda_{i}, \mu_{i}\right)$, and adding two terms proportional to $\frac{\partial f_{\lambda \mu}}{\partial \lambda}$ and $\frac{\partial f_{\lambda \mu}}{\partial \mu}$ respectively. Applying this vectorfield to a coordinate $\lambda_{j}$, with $j \neq i$, one gets:

$$
\begin{aligned}
\left\{f_{(i)}, \lambda_{j}\right\}_{\tilde{P}_{1}}-\lambda_{i}\left\{f_{(i)}, \lambda_{j}\right\}_{P_{0}}= & \left.\left\{f_{\lambda \mu}, \lambda_{j}\right\}_{\left(\tilde{P}_{1}-\lambda P_{0}\right)}\right|_{\lambda_{i}, \mu_{i}}+ \\
& \left.\left(\left\{\lambda_{i}, \lambda_{j}\right\}_{\tilde{P}_{1}}-\lambda_{i}\left\{\lambda_{i}, \lambda_{j}\right\}_{P_{0}}\right) \frac{\partial f_{\lambda \mu}}{\partial \lambda}\right|_{\lambda_{i}, \mu_{i}}+ \\
& \left.\left(\left\{\mu_{i}, \lambda_{j}\right\}_{\tilde{P}_{1}}-\lambda_{i}\left\{\mu_{i}, \lambda_{j}\right\}_{P_{0}}\right) \frac{\partial f_{\lambda \mu}}{\partial \mu}\right|_{\lambda_{i}, \mu_{i}} .
\end{aligned}
$$


By hypothesis, $\lambda_{j}$ is in involution with both $\lambda_{i}$ and $\mu_{i}$ for $j \neq i$; therefore, only the first line survives, but due to (3.14) the r.h.s. is equal to $-\sum_{\alpha=1}^{k} S_{\alpha}\left(\lambda_{i}, \mu_{i}\right)\left\{h^{\alpha}, \lambda_{j}\right\}_{P_{0}}$, which once again vanishes on account of (3.17): half of (3.19) is proved. Repeating the whole argument for the coordinate $\mu_{i}$, upon replacing $\tilde{P}_{1}$ with $\tilde{P}_{2}$, one proves the full statement.

Thus, the search for separation variables is completely translated into the problem of finding a set of "good" transversal vectorfields. We leave three questions open. First, suppose that one were able to find (in some other way) a set of generalised DarbouxNijenhuis coordinates for the "deformed" Poisson triple $\left(P_{0}, \tilde{P}_{1}, \widetilde{P}_{2}\right)$; would these coordinates be roots of the polynomials $S_{\alpha}$ (without imposing further conditions)?

Second question, is requirement (3) in Prop.(3.10) really necessary, or is it already implied by the previous assumptions? We could not find any concrete example in which (3.2) and the requirements (1), (2) of Prop.(3.10) are satisfied, but (3) fails to hold. One could then suspect that the equality (3) can be derived from the other (simpler) assumptions. The trouble with the requirement (3) is that it is both uneasy to check and lacking a clear geometrical significance円; nevertheless, we have not yet succeeded in replacing it with another condition equally ensuring that $\left\{\lambda_{i}, \mu_{i}\right\}_{P_{0}}=1$.

Third open problem: under our assumptions, we have obtained Sklyanin's separation condition with $W_{i}=f_{(i)}-p_{i}\left(\lambda_{i}, \mu_{i}\right)$. In the algebro-geometric setting, one deals with a more particular situation, namely $p_{i}\left(\lambda_{i}, \mu_{i}\right)=p\left(\lambda_{i}, \mu_{i}\right)$ for a fixed polynomial $p(\lambda, \mu)$ not depending on $i$, so all separation variables are (pairwise) roots of a single polynomial $f_{\lambda \mu}-p(\lambda, \mu)$, defining the spectral curve of the system. At the moment, we do not know which additional conditions would ensure this stronger type of separability, which is encountered in the examples that we discuss below. In the next subsection we shall produce an example showing that the occurrence of a single spectral curve does not follow automatically from our assumptions.

\subsection{Canonical form of trihamiltonian structures in separation coordinates}

As we have seen, given a trihamiltonian structure $\left(P_{0}, P_{1}, P_{2}\right)$ and a complete common Casimir polynomial $f_{\lambda \mu}$, one needs to find a good set of transversal vectorfields to produce separation variables. Finding such vectorfields is, in general, a difficult task. On the other hand, upon assuming that such vectorfields exist, one can explicitly compute

\footnotetext{
${ }^{1}$ In principle, it would be possible to consider the manifold $\mathcal{M} \times \mathbb{R}^{2}$ (with the spectral parameters $\lambda, \mu$ regarded as two additional real coordinates), endowed with the direct sum of the structure $P_{0}$ on $\mathcal{M}$ with the Poisson structure on $\mathbb{R}^{2}$ for which $(\lambda, \mu)$ are canonical coordinates. Then, $(3)$ could be rephrased by saying that all the Poisson brackets of the functions $S_{\alpha}$ (regarded as functions of $2 m+k+2$ variables) should vanish on each submanifold described by the equations $\lambda=\lambda_{i}, \mu=\mu_{i}$. Still, this does not seem to help very much.
} 
the components of all the relevant object as they would become in separation coordinates, as we shall see now; in some cases, this provides a concrete procedure to obtain "backwards" the change of variables.

From the previous discussion, we know that in a generalised Darboux-Nijenhuis coordinate system:

1. as far as the $2 m \times 2 m$ block corresponding to the coordinates $\left(\lambda_{i}, \mu_{i}\right)$ is considered, the tensor $P_{0}$ is in canonical form, while the tensors $\tilde{P}_{1}$ and $\tilde{P}_{2}$ are obtained from $P_{0}$ by applying the diagonal recursion operators $N_{1}$ and $N_{2}$, having the coordinates $\lambda_{i}$ and $\mu_{i}$, respectively, as (double) eigenvalues;

2. the components of the complete tensors $P_{0}, \tilde{P}_{1}$ and $\tilde{P}_{2}$ in the coordinates $\left(\lambda_{i}, \mu_{i}, c^{\alpha}\right)$ are obtained by simply adding $k$ null rows and $k$ null columns to the respective $2 m \times 2 m$ matrix (by "null" we mean that all the corresponding entries are vanishing);

3. the transversal vectorfields $Z_{\alpha}$ are coordinate vectorfields: $Z_{\alpha}=\frac{\partial}{\partial c^{\alpha}}$;

4. the relation between the original Poisson tensors $P_{1}$ and $P_{2}$ with the "deformed" ones, $\tilde{P}_{1}$ and $\tilde{P}_{2}$, is given by (3.5);

5. each pair of conjugate coordinates $\left(\lambda_{i}, \mu_{i}\right)$ is a root of the equation $f_{\lambda \mu}-p_{i}(\lambda, \mu)=0$ for some polynomial $p_{i}$.

The latter information allows one to find the explicit expression of all the hamiltonians $h_{j}^{i}$ as functions of $\left(\lambda_{i}, \mu_{i}, c^{\alpha}\right)$, once fixed the polynomials $p_{i}$ (which may be separately determined or arbitrarily chosen, as we explain below). In fact, let us assume as before that the common Casimir polynomial $f_{\lambda \mu}$ contains exactly $m+k$ independent hamiltonians $h_{j}^{i}$. Let us single out the $k$ hamiltonians which are Casimir functions for $P_{0}$, which we denote as above by $c^{\alpha}$, and denote the remaining (independent) hamiltonians as $h_{A}$, with $A=1, \ldots, m$. We impose the conditions

$$
\begin{gathered}
f_{(1)}\left(\lambda_{1}, \mu_{1}\right)=p_{1}\left(\lambda_{1}, \mu_{1}\right) \\
\vdots \\
f_{(m)}\left(\lambda_{m}, \mu_{m}\right)=p_{m}\left(\lambda_{m}, \mu_{m}\right)
\end{gathered}
$$

which form a linear system of $m$ independent equations in the $m$ unknowns $h_{A}$. Solving it, one finds $h_{A}=h_{A}\left(\lambda_{i}, \mu_{i}, c^{\alpha}\right)$.

Next, one produces the deformation vectorfields $X_{P_{1}}$ and $X_{P_{2}}$ according to Lemma (3.7). Then, one can compute the components of the two Poisson tensors $P_{1}$ and $P_{2}$ :

$$
P_{1}=\tilde{P}_{1}-\mathcal{L}_{X_{P_{1}}} P_{0} \quad \text { and } \quad P_{2}=\tilde{P}_{2}-\mathcal{L}_{X_{P_{2}}} P_{0}
$$


It is also important to remark that one can obtain as well the expression of the polynomials $S_{\alpha}(\lambda, \mu)=\frac{\partial f_{\lambda \mu}}{\partial c^{\alpha}}$. This fact will be used in the applications.

To fix the ideas, we work out a concrete example. We remark that in this way we shall display a concrete case where the requirements of Prop.(3.10) can be directly tested, showing that the set of systems fulfilling our requirements is indeed not empty (other examples can be produced in the same way, starting from different "fundamental molecules"). Take the $\mathfrak{g r}(3)$ "fundamental molecule" represented in (1.27). We set $c^{1} \equiv$ $h_{2}^{0}, c^{2} \equiv h_{1}^{1}$ and $c^{3} \equiv h_{0}^{2}$; we also simplify the notation for the remaining hamiltonians by setting $h_{1} \equiv h_{0}^{0}, h_{2} \equiv h_{1}^{0}$, and $h_{3} \equiv h_{0}^{1}$. The common Casimir polynomial (apart from possible constant terms, which may anyhow be compensated in the polynomials $p_{i}$ ) becomes

$$
f_{\lambda \mu}=h_{1}+h_{2} \lambda+c_{1} \lambda^{2}+h_{3} \mu+c_{2} \lambda \mu+c_{3} \lambda \mu^{2} .
$$

We leave the three polynomials $p_{1}, p_{2}$ and $p_{3}$ undetermined; for brevity, we write $p_{(1)}$ for $p_{1}\left(\lambda_{1}, \mu_{1}\right)$, and so on. Imposing (3.20), the hamiltonians read as follows:

$$
\begin{aligned}
& h_{1}=[\left(\lambda_{2} \mu_{3}-\lambda_{3} \mu_{2}\right) p_{(1)}+\left(\lambda_{3} \mu_{1}-\lambda_{1} \mu_{3}\right) p_{(2)}+\left(\lambda_{1} \mu_{2}-\lambda_{2} \mu_{1}\right) p_{(3)}+ \\
& {\left[\left(\lambda_{3}-\lambda_{2}\right) \lambda_{2} \lambda_{3} \mu_{1}+\left(\lambda_{1}-\lambda_{3}\right) \lambda_{1} \lambda_{3} \mu_{2}+\left(\lambda_{2}-\lambda_{1}\right) \lambda_{1} \lambda_{2} \mu_{3}\right] c_{1}+} \\
& {\left[\left(\mu_{3}-\mu_{2}\right) \lambda_{2} \lambda_{3} \mu_{1}+\left(\mu_{1}-\mu_{3}\right) \lambda_{1} \lambda_{3} \mu_{2}+\left(\mu_{2}-\mu_{1}\right) \lambda_{1} \lambda_{2} \mu_{3}\right] c_{2}+} \\
& {\left.\left[\left(\mu_{2}-\mu_{3}\right) \mu_{2} \mu_{3} \lambda_{1}+\left(\mu_{3}-\mu_{1}\right) \mu_{1} \mu_{3} \lambda_{2}+\left(\mu_{1}-\mu_{2}\right) \mu_{1} \mu_{2} \lambda_{3}\right] c_{3}\right] . } \\
& \cdot\left(\lambda_{1} \mu_{2}-\lambda_{2} \mu_{1}+\lambda_{2} \mu_{3}-\lambda_{3} \mu_{2}+\lambda_{3} \mu_{1}-\lambda_{1} \mu_{3}\right)^{-1} \\
& h_{2}=[\left(\mu_{2}-\mu_{3}\right) p_{(1)}+\left(\mu_{3}-\mu_{1}\right) p_{(2)}+\left(\mu_{1}-\mu_{2}\right) p_{(3)}+ \\
& {\left[\left(\lambda_{2}{ }^{2}-\lambda_{3}^{2}\right) \mu_{1}+\left(\lambda_{3}{ }^{2}-\lambda_{1}{ }^{2}\right) \mu_{2}+\left(\lambda_{1}{ }^{2}-\lambda_{2}{ }^{2}\right) \mu_{3}\right] c_{1}+} \\
& {\left[\left(\lambda_{2}-\lambda_{1}\right) \mu_{1} \mu_{2}+\left(\lambda_{1}-\lambda_{3}\right) \mu_{1} \mu_{3}+\left(\lambda_{3}-\lambda_{2}\right) \mu_{2} \mu_{3}\right] c_{2}+} \\
& {\left.\left[\left(\mu_{2}-\mu_{1}\right) \mu_{1} \mu_{2}+\left(\mu_{1}-\mu_{3}\right) \mu_{1} \mu_{3}+\left(\mu_{3}-\mu_{2}\right) \mu_{2} \mu_{3}\right] c_{3}\right] . } \\
& \cdot\left(\lambda_{1} \mu_{2}-\lambda_{2} \mu_{1}+\lambda_{2} \mu_{3}-\lambda_{3} \mu_{2}+\lambda_{3} \mu_{1}-\lambda_{1} \mu_{3}\right)^{-1} \\
&\left(\lambda_{3}-\lambda_{2}\right) p_{(1)}+\left(\lambda_{3}-\lambda_{2}\right) p_{(1)}+\left(\lambda_{3}-\lambda_{2}\right) p_{(1)}+ \\
& h_{3}=\left[\left(\lambda_{1}-\lambda_{2}\right) \lambda_{1} \lambda_{2}+\left(\lambda_{3}-\lambda_{1}\right) \lambda_{1} \lambda_{3}+\left(\lambda_{2}-\lambda_{3}\right) \lambda_{2} \lambda_{3}\right] c_{1}+ \\
& {\left[\left(\mu_{1}-\mu_{2}\right) \lambda_{1} \lambda_{2}+\left(\mu_{3}-\mu_{1}\right) \lambda_{1} \lambda_{3}+\left(\mu_{2}-\mu_{3}\right) \lambda_{2} \lambda_{3}\right] c_{2}+} \\
& {\left.\left[\left(\mu_{3}{ }^{2}-\mu_{2}{ }^{2}\right) \lambda_{1}+\left(\mu_{1}{ }^{2}-\mu_{3}{ }^{2}\right) \lambda_{2}+\left(\mu_{2}{ }^{2}-\mu_{1}{ }^{2}\right) \lambda_{3}\right] c_{3}\right] . } \\
& \cdot\left(\lambda_{1} \mu_{2}-\lambda_{2} \mu_{1}+\lambda_{2} \mu_{3}-\lambda_{3} \mu_{2}+\lambda_{3} \mu_{1}-\lambda_{1} \mu_{3}\right)^{-1}
\end{aligned}
$$


The deformation vectorfields (3.6) are

$$
X_{P_{1}}=h_{2} \frac{\partial}{\partial c^{1}}+h_{3} \frac{\partial}{\partial c^{2}}, \quad X_{P_{2}}=h_{2} \frac{\partial}{\partial c^{2}}+h_{3} \frac{\partial}{\partial c^{3}} .
$$

Starting from

$$
P_{0}=\left(\begin{array}{ccccccccc}
0 & 0 & 0 & 1 & 0 & 0 & 0 & 0 & 0 \\
0 & 0 & 0 & 0 & 1 & 0 & 0 & 0 & 0 \\
0 & 0 & 0 & 0 & 0 & 1 & 0 & 0 & 0 \\
-1 & 0 & 0 & 0 & 0 & 0 & 0 & 0 & 0 \\
0 & -1 & 0 & 0 & 0 & 0 & 0 & 0 & 0 \\
0 & 0 & -1 & 0 & 0 & 0 & 0 & 0 & 0 \\
0 & 0 & 0 & 0 & 0 & 0 & 0 & 0 & 0 \\
0 & 0 & 0 & 0 & 0 & 0 & 0 & 0 & 0 \\
0 & 0 & 0 & 0 & 0 & 0 & 0 & 0 & 0
\end{array}\right)
$$

$\tilde{P}_{1}=\left(\begin{array}{ccccccccc}0 & 0 & 0 & \lambda_{1} & 0 & 0 & 0 & 0 & 0 \\ 0 & 0 & 0 & 0 & \lambda_{2} & 0 & 0 & 0 & 0 \\ 0 & 0 & 0 & 0 & 0 & \lambda_{3} & 0 & 0 & 0 \\ -\lambda_{1} & 0 & 0 & 0 & 0 & 0 & 0 & 0 & 0 \\ 0 & -\lambda_{2} & 0 & 0 & 0 & 0 & 0 & 0 & 0 \\ 0 & 0 & -\lambda_{3} & 0 & 0 & 0 & 0 & 0 & 0 \\ 0 & 0 & 0 & 0 & 0 & 0 & 0 & 0 & 0 \\ 0 & 0 & 0 & 0 & 0 & 0 & 0 & 0 & 0 \\ 0 & 0 & 0 & 0 & 0 & 0 & 0 & 0 & 0\end{array}\right) \quad \tilde{P}_{2}=\left(\begin{array}{ccccccccccc}0 & 0 & 0 & \mu_{1} & 0 & 0 & 0 & 0 & 0 \\ 0 & 0 & 0 & \mu_{2} & 0 & 0 & 0 & 0 \\ 0 & 0 & 0 & \mu_{3} & 0 & 0 & 0 \\ 0 & 0 & 0 & 0 & 0 & 0 & 0 & 0 \\ -\mu_{1} & 0 & 0 & 0 \\ 0 & -\mu_{2} & 0 & 0 & 0 & 0 & 0 & 0 \\ 0 & -\mu_{3} & 0 & 0 & 0 & 0 & 0 & 0 \\ 0 & 0 & 0 & 0 & 0 & 0 & 0 & 0 \\ 0 & 0 & 0 & 0 \\ 0 & 0 & 0 & 0 & 0 & 0 \\ 0 & 0 & 0 & 0 & 0 & 0 & 0 & 0 & 0\end{array}\right)$

one can obtain the matrix expressions for the tensors $P_{1}=\tilde{P}_{1}-\mathcal{L}_{X_{P_{1}}} P_{0}$ and $P_{2}=$ $\tilde{P}_{2}-\mathcal{L}_{X_{P_{2}}} P_{0}$. They coincide with the latter two matrices above, respectively, as far as the $6 \times 6$ upper left blocks are concerned, while the remaining three rows and three columns are rather complicated for both tensors (and it would be pointless to write them down here). The fact that the tensors $P_{0}, \tilde{P}_{1}$ and $\tilde{P}_{2}$ above are pairwise compatible Poisson tensors, as well as the fact that the coordinate vecorfields $Z_{\alpha}=\frac{\partial}{\partial c^{\alpha}}$ are symmetries of all of them, are trivially verified; then, is it enough to reverse the steps of the proof of Prop.(3.6) to show that $P_{1}$ and $P_{2}$ are both Poisson tensors compatible with $P_{0}$. One can then check directly that the function (3.22) with the three hamiltonians computed above is a Casimir function for $P_{0}, P_{1}$ and $P_{2}$, as expected.

The polynomials $S_{\alpha}\left(\lambda, \mu ; \lambda_{i}, \mu_{i}\right)$ are

$$
\begin{aligned}
S_{1}= & \frac{\left(\lambda_{3}-\lambda_{2}\right) \lambda_{2} \lambda_{3} \mu_{1}+\left(\lambda_{1}-\lambda_{3}\right) \lambda_{1} \lambda_{3} \mu_{2}+\left(\lambda_{2}-\lambda_{1}\right) \lambda_{1} \lambda_{2} \mu_{3}}{\lambda_{1} \mu_{2}-\lambda_{2} \mu_{1}+\lambda_{2} \mu_{3}-\lambda_{3} \mu_{2}+\lambda_{3} \mu_{1}-\lambda_{1} \mu_{3}}+ \\
& \left(\frac{\left(\lambda_{2}{ }^{2}-\lambda_{3}^{2}\right) \mu_{1}+\left(\lambda_{3}{ }^{2}-\lambda_{1}{ }^{2}\right) \mu_{2}+\left(\lambda_{1}{ }^{2}-\lambda_{2}{ }^{2}\right) \mu_{3}}{\lambda_{1} \mu_{2}-\lambda_{2} \mu_{1}+\lambda_{2} \mu_{3}-\lambda_{3} \mu_{2}+\lambda_{3} \mu_{1}-\lambda_{1} \mu_{3}}\right) \lambda+ \\
& \left(\frac{\left(\lambda_{1}-\lambda_{2}\right) \lambda_{1} \lambda_{2}+\left(\lambda_{3}-\lambda_{1}\right) \lambda_{1} \lambda_{3}+\left(\lambda_{2}-\lambda_{3}\right) \lambda_{2} \lambda_{3}}{\lambda_{1} \mu_{2}-\lambda_{2} \mu_{1}+\lambda_{2} \mu_{3}-\lambda_{3} \mu_{2}+\lambda_{3} \mu_{1}-\lambda_{1} \mu_{3}}\right) \mu+\lambda^{2} \\
S_{2}= & \frac{\left(\mu_{3}-\mu_{2}\right) \lambda_{2} \lambda_{3} \mu_{1}+\left(\mu_{1}-\mu_{3}\right) \lambda_{1} \lambda_{3} \mu_{2}+\left(\mu_{2}-\mu_{1}\right) \lambda_{1} \lambda_{2} \mu_{3}}{\lambda_{1} \mu_{2}-\lambda_{2} \mu_{1}+\lambda_{2} \mu_{3}-\lambda_{3} \mu_{2}+\lambda_{3} \mu_{1}-\lambda_{1} \mu_{3}}+ \\
& \left(\frac{\left(\lambda_{2}-\lambda_{1}\right) \mu_{1} \mu_{2}+\left(\lambda_{1}-\lambda_{3}\right) \mu_{1} \mu_{3}+\left(\lambda_{3}-\lambda_{2}\right) \mu_{2} \mu_{3}}{\lambda_{1} \mu_{2}-\lambda_{2} \mu_{1}+\lambda_{2} \mu_{3}-\lambda_{3} \mu_{2}+\lambda_{3} \mu_{1}-\lambda_{1} \mu_{3}}\right) \lambda+ \\
& \left(\frac{\left(\mu_{1}-\mu_{2}\right) \lambda_{1} \lambda_{2}+\left(\mu_{3}-\mu_{1}\right) \lambda_{1} \lambda_{3}+\left(\mu_{2}-\mu_{3}\right) \lambda_{2} \lambda_{3}}{\lambda_{1} \mu_{2}-\lambda_{2} \mu_{1}+\lambda_{2} \mu_{3}-\lambda_{3} \mu_{2}+\lambda_{3} \mu_{1}-\lambda_{1} \mu_{3}}\right) \mu+\lambda \mu
\end{aligned}
$$




$$
\begin{aligned}
S_{3}= & \frac{\left(\mu_{2}-\mu_{3}\right) \mu_{2} \mu_{3} \lambda_{1}+\left(\mu_{3}-\mu_{1}\right) \mu_{1} \mu_{3} \lambda_{2}+\left(\mu_{1}-\mu_{2}\right) \mu_{1} \mu_{2} \lambda_{3}}{\lambda_{1} \mu_{2}-\lambda_{2} \mu_{1}+\lambda_{2} \mu_{3}-\lambda_{3} \mu_{2}+\lambda_{3} \mu_{1}-\lambda_{1} \mu_{3}}+ \\
& \left(\frac{\left(\mu_{2}-\mu_{1}\right) \mu_{1} \mu_{2}+\left(\mu_{1}-\mu_{3}\right) \mu_{1} \mu_{3}+\left(\mu_{3}-\mu_{2}\right) \mu_{2} \mu_{3}}{\lambda_{1} \mu_{2}-\lambda_{2} \mu_{1}+\lambda_{2} \mu_{3}-\lambda_{3} \mu_{2}+\lambda_{3} \mu_{1}-\lambda_{1} \mu_{3}}\right) \lambda+ \\
& \left(\frac{\left(\mu_{3}^{2}-\mu_{2}^{2}\right) \lambda_{1}+\left(\mu_{1}^{2}-\mu_{3}^{2}\right) \lambda_{2}+\left(\mu_{2}^{2}-\mu_{1}^{2}\right) \lambda_{3}}{\lambda_{1} \mu_{2}-\lambda_{2} \mu_{1}+\lambda_{2} \mu_{3}-\lambda_{3} \mu_{2}+\lambda_{3} \mu_{1}-\lambda_{1} \mu_{3}}\right) \mu+\mu^{2}
\end{aligned}
$$

This set of polynomials satisfies all the conditions of Prop.(3.10): first, they do not depend any more on the coordinates $c^{\alpha}$; second, the reader can check straightforwardly that $\left(\lambda_{1}, \mu_{1}\right),\left(\lambda_{2}, \mu_{2}\right)$ and $\left(\lambda_{3}, \mu_{3}\right)$ are pairs of common roots of the polynomials $S_{1}, S_{2}$ and $S_{3}$; as far as the third condition is concerned, one should check that for any $i=1,2,3$ the two matrices $\left\|\left\{S_{\alpha}, S_{\beta}\right\}_{P_{0}}\right\|_{\lambda_{i}, \mu_{i}}$ and $\left\|\frac{\partial S_{\alpha}}{\partial \lambda} \frac{\partial S_{\beta}}{\partial \mu}-\frac{\partial S_{\alpha}}{\partial \mu} \frac{\partial S_{\beta}}{\partial \lambda}\right\|_{\lambda_{i}, \mu_{i}}$ (with $\alpha, \beta=1,2,3$ ) are not identically vanishing and coincide. Direct computation shows that this is indeed the case: for $i=1$, the two matrices are both equal to

$$
\left(\begin{array}{ccc}
0 & \left(\lambda_{2}-\lambda_{1}\right)\left(\lambda_{3}-\lambda_{1}\right) & \left(\lambda_{2}-\lambda_{1}\right)\left(\mu_{3}-\mu_{1}\right)+ \\
+\left(\mu_{2}-\mu_{1}\right)\left(\lambda_{3}-\lambda_{1}\right) \\
\left(\lambda_{1}-\lambda_{2}\right)\left(\lambda_{3}-\lambda_{1}\right) & 0 & \left(\mu_{2}-\mu_{1}\right)\left(\mu_{3}-\mu_{1}\right) \\
& & \\
\left(\lambda_{1}-\lambda_{2}\right)\left(\mu_{3}-\mu_{1}\right)+ & \left(\mu_{1}-\mu_{2}\right)\left(\mu_{3}-\mu_{1}\right) & 0 \\
+\left(\mu_{1}-\mu_{2}\right)\left(\lambda_{3}-\lambda_{1}\right) &
\end{array}\right)
$$

and similar expressions, with the appropriate permutiations of indices, are found for $i=2,3$.

It is worthwhile to remark that one can produce, choosing arbitrarily the polynomials $p_{i}(\lambda, \mu)$, infinitely many families of hamiltonians which are separable according to Sklyanin's criterion, but do not coincide with the coefficients of a single spectral curve, unless one sets $p_{1}(\lambda, \mu) \equiv p_{2}(\lambda, \mu) \equiv p_{3}(\lambda, \mu) \equiv p(\lambda, \mu)$.

Notice that the choice of the constant polynomials $p_{i}$ does not affect the polynomials $S_{\alpha}$. Actually, if one starts from a fixed trihamiltonian structure and is able to find vectorfields $Z_{\alpha}$ fulfilling all the requirement listed in Prop.(3.10), then the polynomials $p_{i}$ are determined a posteriori simply by plugging separately each pair of common roots of the polynomials $S_{\alpha}$ into $f_{\lambda \mu}$. The above reconstruction of the trihamiltonian structure goes in the reverse direction: the polynomials $p_{i}$ are arbitrary and determine at the same time the hamiltonians and the Poisson tensors $P_{1}$ and $P_{2}$.

Thus, in our framework the constant terms in Sklyanin's separation polynomials $W_{i}$, and a fortiori in the spectral curve (whenever it exists), are not directly encoded in the hamiltonian structure underlying a dynamical system and its symmetries (some aspects connected to the arbitrarity of the constant part of the the spectral curve equation have been addressed by J. Harnad in [23]). We have seen that the constant polynomials 
$p_{i}$ are determined by the choice of a set of transversal vectorfields, or equivalently of a system of separation variables: possible different sets of polynomials $p_{i}-$ and, eventually, different spectral curves - are associated to different sets of separation variables. Indeed, from the example given above one might infer that different choices of the polynomials $p_{i}$ lead to different hamiltonians (hence, to distinct dynamical systems), but in fact these are - by construction - nothing but the same hamiltonians expressed in two different coordinate systems: the same holds for the components of both $P_{1}$ and $P_{2}$. The transversal vectorfields, on the contrary, would have the same components in both coordinate systems, but the vectorfields themselves would not be the same, as happens for the deformed structures $\tilde{P}_{1}$ and $\tilde{P}_{2}$.

In conclusion, the spectral curve appears to be an additional datum with respect to the purely hamiltonian structure of an integrable system; its hamiltonian interpretation, however, is deeply connected to the existence of particular canonical coordinates, as (separately) suggested by Sklyanin and Magri. For trihamiltonian structures (with a suitable projection onto a symplectic leaf), we have found a sound connection between separation coordinates and the vanishing of spectral polynomials.

\section{Separation coordinates for Lax equations with spec- tral parameter}

In this section we apply the techniques discussed so far to a particular class of dynamical systems, represented by Lax equations with spectral parameter. More precisely, we shall restrict to the following situation:

1. the Lax operator is a $r \times r$ matrix polynomial of degree $n$ in the spectral parameter, $L(\lambda)=A \lambda^{n}+M_{1} \lambda^{n-1}+\cdots+M_{n}$, with a constant leading term $A \in \mathfrak{g l}(r) ;$

2. the constant matrix $A$ should commute only with linear combinations of its powers (including $A^{0} \equiv \mathbb{I}$ ). Equivalently, if $A$ is diagonalisable, it should have distinct eigenvalues; more generally, the canonical Jordan form of $A$ should not contain Jordan blocks proportional to the identity of dimension higher than one. This property is generic on $\mathfrak{g l}(r)$, but the requirement rules out some cases considered in the literature [2] [3]. A consequence of this requirement is that $A$ may be nilpotent, with $A^{r}=0$, but $A^{k}$ should not vanish for $k<r$.

3. the variable matrices $M_{i}$ are generic matrices belonging to $\mathfrak{g l}(r)$ : we are not considering restrictions to proper subalgebras, nor other types of reductions.

This setting includes classical models such as the Euler-Poinsot top (for $r=3, n=1$ ) and the Lagrange top (for $r=3, n=2$ ), in the sense which has already been explained in the introduction: the classical models are properly embedded into larger systems, 
but can be recovered by simple restriction (of a subset of the flows) on the appropriate invariant submanifold (namely, the subalgebra of antisymmetric matrices). Other important examples such as the Kovalewska top, or the Dubrovin-Novikov finite-dimensional reductions [18] of the Gel'fand-Dickey soliton hierarchies, are strictly related to the systems that we are considering but cannot be directly obtained by simple restriction " $a$ posteriori". The application of our framework to the periodic Toda lattice or to other models without a constant leading term in the Lax matrix has not been investigated yet.

There are two possible approaches to the construction of multihamiltonian structures for Lax equations of the type considered. Most authors regard them as dynamical systems on loop algebras $\tilde{\mathfrak{g l}}(r) \equiv \mathfrak{g l}(r)((\lambda))$, and use the $R$-matrix technique to define compatible Poisson brackets, which can be reduced to finite-dimensional quotient spaces identified with the linear spaces of fixed-order polynomials in $\lambda$ [21].

According to another approach, one considers the direct sum of $n$ copies of the Lie algebra $\mathfrak{g l}(r)$, defines a suitable Lie algebra structure on this vector space (different from the direct product structure), and an appropriate scalar product; in this way, one gets a natural Lie-Poisson bracket on $\mathfrak{g l}(r)^{n}$. The other Poisson structures are obtained by a deformation procedure, i.e. are defined as Lie derivatives of the LiePoisson tensor along suitable vectorfields [11]. In this approach, the dynamical variable is a $n$-ple of matrices $\left(M_{1}, \ldots, M_{n}\right)$, while the fixed matrix $A$ occurs in the definition of the deformation vectorfields which produce the Poisson structures; the Lax matrix $L(\lambda)$ itself arises as a by-product, in connection with the Hamilton equations with spectral parameter which naturally represent the trihamiltonian flows. The two approaches are substantially equivalent for our purposes. We shall follow here the second approach, but we stress that most of the definitions could be rephrased in the $R$-matrix language.

As anticipated in the introduction, we are not giving here all the proofs. The proof that the tensors $P_{0}$ and $P_{1}$ defined below are compatible Poisson tensors can be found, for instance, in [11] or [21]. The only proof which is included concerns the fact that the characteristic determinant of the Lax matrix is the fundamental common Casimir polynomial for our trihamiltonian structure.

\subsection{Affine Lie-Poisson pencils}

Let us consider the linear space $\mathfrak{g l}(r)^{n} \equiv \bigoplus^{n} \mathfrak{g l}(r)$; we shall denote elements of this space by $\mathbf{M} \equiv\left(M_{1}, \ldots, M_{n}\right)$, with $M_{i} \in \mathfrak{g l}(r)$. The scalar product on $\mathfrak{g l}(r)$ defined by (1.5) is extended "componentwise" to $\mathfrak{g l}(r)^{n}$ :

$$
(\mathbf{A}, \mathbf{B})=\sum_{i=1}^{n} \operatorname{Tr}\left(A_{i} \cdot B_{i}\right) .
$$

Using this scalar product, the gradient of a function $f: \mathfrak{g l}(r)^{n} \rightarrow \mathbb{R}$ is again an element of $\mathfrak{g l}(r)^{n}$, that we denote by $\left(\nabla_{1} f, \ldots, \nabla_{n} f\right)$. We define on $\mathfrak{g l}(r)^{n}$ a first Lie-Poisson 
structure $P^{(n)}$ (depending on $M_{i}$ in a strictly linear way) by setting

$$
\{f, g\}_{P^{(n)}}=\sum_{i=1}^{n}\left(\nabla_{i} g, \sum_{k=i}^{n}\left[M_{k}, \nabla_{k-i+1} f\right]\right) .
$$

It is convenient to represent the Poisson tensor $P^{(n)}$ as a matrix of linear operators acting on the column vector $\left(\nabla_{1} f, \ldots, \nabla_{n} f\right)$ :

$$
P^{(n)}=\left(\begin{array}{ccccc}
{\left[M_{1}, \cdot\right]} & {\left[M_{2}, \cdot\right]} & \ldots & {\left[M_{n-1}, \cdot\right]} & {\left[M_{n}, \cdot\right]} \\
{\left[M_{2}, \cdot\right]} & {\left[M_{3}, \cdot\right]} & \ldots & {\left[M_{n}, \cdot\right]} & 0 \\
\vdots & \vdots & & \vdots & \vdots \\
{\left[M_{n-1}, \cdot\right]} & {\left[M_{n}, \cdot\right]} & \ldots & 0 & 0 \\
{\left[M_{n}, \cdot\right]} & 0 & \ldots & 0 & 0
\end{array}\right)
$$

Following [11], from this first structure it is possible to obtain a sequence of other $n$ affine Poisson structures $P^{(n-1)}, \ldots, P^{(0)}$, all mutually compatible, by the iterative formula

$$
P^{(k-1)}=-\frac{1}{k+1} \mathcal{L}_{X} P^{(k)} \quad k=n \ldots 1
$$

where the components of the deformation vectors field $X$ are affine functions, determined by a the fixed matrix $A \in \mathfrak{g l}(r)$ :

$$
X=\left(\begin{array}{ccccc}
0 & \cdots & \cdots & \cdots & 0 \\
n-1 & \ddots & & & \vdots \\
0 & \ddots & \ddots & & \vdots \\
\vdots & \ddots & \ddots & \ddots & \vdots \\
0 & \cdots & 0 & 1 & 0
\end{array}\right)\left(\begin{array}{c}
M_{1} \\
\vdots \\
\vdots \\
\vdots \\
M_{n}
\end{array}\right)+\left(\begin{array}{c}
n A \\
0 \\
\vdots \\
\vdots \\
0
\end{array}\right)
$$

The first pencil of the trihamiltonian structure that we shall consider is defined by the tensors $P^{(1)}$ and $P^{(0)}$ of the sequence; their expression is

$$
\begin{aligned}
\{f, g\}_{P^{(1)}}= & \left(\nabla_{1} g,\left[\nabla_{n-1} f, A\right]\right)+\sum_{i=2}^{n-1}\left(\nabla_{i} g,\left[\nabla_{n-i} f, A\right]+\sum_{k=1}^{i-1}\left[\nabla_{n-i+k} f, M_{k}\right]\right) \\
& +\left(\nabla_{n} g,\left[M_{n}, \nabla_{n} f\right]\right) \\
\{f, g\}_{P^{(0)}}= & \left(\nabla_{1} g,\left[\nabla_{n} f, A\right]\right)+\sum_{i=2}^{n}\left(\nabla_{i} g,\left[\nabla_{n-i+1} f, A\right]+\sum_{k=1}^{i-1}\left[\nabla_{n-i+k+1} f, M_{k}\right]\right) .
\end{aligned}
$$


In matrix representation,

$$
\begin{aligned}
P^{(1)}= & \left(\begin{array}{ccccc}
0 & \ldots & 0 & {[\cdot, A]} & 0 \\
0 & \cdots & {[\cdot, A]} & {\left[\cdot, M_{1}\right]} & 0 \\
\vdots & & & \vdots & 0 \\
0 & {[\cdot, A]} & \ldots & {\left[\cdot, M_{n-3}\right]} & 0 \\
{[\cdot, A]} & {\left[\cdot, M_{1}\right]} & \ldots & {\left[\cdot, M_{n-2}\right]} & 0 \\
0 & \cdots & \ldots & 0 & {\left[M_{n}, \cdot\right]}
\end{array}\right) \\
P^{(0)}= & \left(\begin{array}{ccccc}
0 & \ldots & \ldots & 0 & {[\cdot, A]} \\
0 & \cdots & \ldots & {[\cdot, A]} & {\left[\cdot, M_{1}\right]} \\
\vdots & & & \vdots & \vdots \\
0 & {[\cdot, A]} & \ldots & & {\left[\cdot, M_{n-2}\right]} \\
{[\cdot, A]} & {\left[\cdot, M_{1}\right]} & \ldots & & {\left[\cdot, M_{n-1}\right]}
\end{array}\right)
\end{aligned}
$$

The Poisson pencil $P^{(1)}-\lambda P^{(0)}$ is called the affine Lie-Poisson pencil on $\mathfrak{g l}(r)$. From the matrix representation given above, it is easy to see that any function $f_{\lambda}=\sum h_{i} \lambda^{i}$ such that

$$
\left\{\begin{aligned}
\nabla_{k} f_{\lambda}-\lambda \nabla_{k+1} f_{\lambda} & =0, \quad k=1, \ldots, n-1 \\
{\left[A \lambda^{n}+M_{1} \lambda^{n-1}+\cdots+M_{n}, \nabla_{n} f_{\lambda}\right] } & =0 .
\end{aligned}\right.
$$

is a Casimir function of the affine Lie-Poisson pencil. This observation leads to the definition of the Lax matrix $L(\lambda)=A \lambda^{n}+M_{1} \lambda^{n-1}+\cdots+M_{n}$, and one immediately sees that the trace of any power of $L(\lambda)$ fulfills (4.5). Each Casimir function of this type generate a bihamiltonian hierarchy according to the prescriptions (1.19) and (1.20). The corresponding Hamilton equations with spectral parameter are equivalent to Lax equations for $L(\lambda)$ : the $k$-th flow of the hierarchy, using the same notation as in (1.19), is represented by

$$
\dot{L}(\lambda)=\left[L(\lambda), \nabla_{n} f_{\lambda}^{(k)}\right] .
$$

In the sequel, we revert to the notation used in the previous part of the paper, setting $P_{0} \equiv P^{(0)}$ and $P_{1} \equiv P^{(1)}$. However, on $\mathfrak{g l}(r)^{n}$ the third compatible structure $P_{2}$, necessary to construct the appropriate second pencil, does not belong to the abovedefined sequence of affine Lie-Poisson tensors: in particular, it has nothing to do with the structure $P^{(2)}$, defined by (4.3) for $n \geq 2$ (for this reason we had to adopt a different notation).

Our method to find the third Poisson tensor $P_{2}$ still starts from the linear Lie-Poisson structure (4.2), but instead of applying a deformation vectorfield we exploit the LaxNijenhuis equation. This equation has been introduced in [5] in connection with the following question: "Given a Lax equation, and a Poisson structure $P$ for which the 
traces of the powers of the Lax matrix are in involution, does it exists a second compatible Poisson structure $Q$ such that the same constants of motion are iteratively linked in a Magri-Lenard hierarchy?" It turns out that, if such a second Poisson structure exists, the two derivatives of the Lax matrix $L$ along the two vectorfields $P d h$ and $Q d h$ generated by any hamiltonian $h$ are linked by the following relation:

$$
\mathcal{L}_{Q d h} L=\frac{1}{2} \mathcal{L}_{P d h}\left(L^{2}\right)+[L, \alpha(d h)]
$$

for some matrix $\alpha$ algebraically depending on differential of the hamiltonian $h$. In some cases, this equation allows one to determine completely the second Poisson structure $Q$. This may happen if the Lax matrix is not generic, and in particular if $L$ is assumed to have a fixed degree with respect to some grading in the Lie algebra; for instance, when $L$ is tridiagonal (Toda), or is a polynomial of fixed degree in a spectral parameter (the case we are dealing with). Then, its square $L^{2}$ has usually a different degree, and the unknown element $\alpha$ occurring in the r.h.s. become determined by a compatibility requirement, i.e. its commutator with $L$ should cancel exactly the terms of higher degree in the derivative of $L^{2}$. We refer the reader to [5] for a complete presentation of the method.

We omit the details of the computation in our case: a key point is that one should plug in the Lax-Nijenhuis a slightly modified Lax matrix polynomial, namely the "convoluted" polynomial $L^{*}(\lambda)=A+M_{1} \lambda+\cdots+M_{n} \lambda^{n}$. Then, starting from the Poisson tensor $P_{0}$, one finds the following new Poisson bracket:

$$
\begin{aligned}
\{f, g\}_{P_{2}}= & \sum_{i=1}^{n}\left(\nabla_{i} g, \sum_{k=i}^{n} M_{k} \nabla_{k-i+1} f A-A \nabla_{k-i+1} f M_{k}\right)+ \\
& \sum_{i=2}^{n}\left(\nabla_{i} g, \sum_{k=i}^{n} \sum_{l=1}^{i-1} M_{k} \nabla_{k+l-i+1} f M_{l}-M_{l} \nabla_{k+l-i+1} f M_{k}\right) .
\end{aligned}
$$

For $n$ generic, writing down the representation of this Poisson tensor as a matrix of linear operators, analogous to (4.4), would be rather cumbersome. The reader can easily figure out the general form from the representations of the Poisson tensors respectively corresponding to $n=1,2,3$ :

$$
\begin{aligned}
& \text { for } n=1, \quad P_{2}=M(\cdot) A-A(\cdot) M \\
& \text { for } n=2, \quad P_{2}=\left(\begin{array}{cc}
M_{1}(\cdot) A-A(\cdot) M_{1} & M_{2}(\cdot) A-A(\cdot) M_{2} \\
M_{2}(\cdot) A-A(\cdot) M_{2} & M_{2}(\cdot) M_{1}-M_{1}(\cdot) M_{2}
\end{array}\right)
\end{aligned}
$$




$$
\text { for } n=3, \quad P_{2}=\left(\begin{array}{ccc}
M_{1}(\cdot) A-A(\cdot) M_{1} & M_{2}(\cdot) A-A(\cdot) M_{2} & M_{3}(\cdot) A-A(\cdot) M_{3} \\
& & \\
M_{2}(\cdot) A-A(\cdot) M_{2} & M_{3}(\cdot) A-A(\cdot) M_{3}+ & M_{2}(\cdot) M_{1}-M_{1}(\cdot) M_{2} \\
& M_{3}-M_{1}(\cdot) M_{3} \\
M_{3}(\cdot) A-A(\cdot) M_{3} & M_{3}(\cdot) M_{1}-M_{1}(\cdot) M_{3} & M_{3}(\cdot) M_{2}-M_{2}(\cdot) M_{3}
\end{array}\right)
$$

For $n=1$ one recovers the Poisson structure of Morosi and Pizzocchero 15 already described in the Introduction. The quadratic Poisson structures for $n>1$ have never been presented in the previous literature, to our knowledge. In the $R$-matrix language, they can be obtained by a suitable modification of the so-called Sklyanin bracket [20].

\subsection{Fundamental Casimir polynomial}

We have anticipated in the introduction that the characteristic determinant of the Lax matrix $L(\lambda)$ provides a complete common Casimir polynomial for the two pencils $P_{1}-\lambda P_{0}$ and $P_{2}-\mu P_{0}$. We shall now prove this statement.

We already know that the traces of the powers of the Lax matrix are Casimir functions for the first pencil, as they fulfill (4.5). The same holds for the coefficients of each power of $\mu$ in the characteristic polynomial $f_{\lambda \mu}=\operatorname{det}(L(\lambda)-\mu \mathbb{I})$, since these coefficients are functionally dependent on the traces of the powers of $L(\lambda)$. Then, what remains to check is that the polynomials in $\mu$ which occur as coefficients of each power of $\lambda$ in $f_{\lambda \mu}$ are Casimir functions for the second pencil.

In the following computation, we denote the components of the gradient of $f_{\lambda \mu}$ by $V_{i}=\nabla_{i} f_{\lambda, \mu}$ (notice that each matrix $V_{i}$ still depends on both $\lambda$ and $\mu$ ), and we systematically use the fact that

$$
V_{k}=\lambda V_{k+1}, \quad \text { i.e. } \quad V_{k}=\lambda^{n-k} V_{n} .
$$

The condition $\left(P_{2}-\mu P_{0}\right) d f_{\lambda \mu}=0$ translates into the following system of $n$ equations:

$$
\left\{\begin{array}{l}
{\left[\mu V_{n}, A\right]-\sum_{k=1}^{n}\left(M_{k} V_{k} A-A V_{k} M_{k}\right)=0} \\
{\left[\mu V_{n-i+1}, A\right]+\sum_{k=1}^{i-1}\left[\mu V_{n-i+k+1}, M_{k}\right]} \\
-\sum_{k=i}^{n}\left(M_{k} V_{k-i+1} A-A V_{k-i+1} M_{k}\right) \\
-\sum_{k=i}^{n} \sum_{l=1}^{i-1}\left(M_{k} V_{k+l-i+1} M_{l}-M_{l} V_{k+l-i+1} M_{k}\right)=0 \text { for } i=2, \ldots, n
\end{array}\right.
$$

which, with some algebraic manipulations taking account of (4.8) and of some of the equalities following from $\left(P_{1}-\lambda P_{0}\right) d f_{\lambda \mu}=0$, can be shown to be equivalent to

$$
(L(\lambda)-\mu \mathbb{I}) V_{n} M_{i}=M_{i} V_{n}(L(\lambda)-\mu \mathbb{I}) \quad \text { for all } i=1 \ldots, n
$$

Now, for the characteristic polynomial $f_{\lambda \mu}=\operatorname{det}(L(\lambda)-\mu \mathbb{I})$ one has

$$
V_{n}=\nabla_{n} f_{\lambda \mu}=f_{\lambda \mu} \cdot(L(\lambda)-\mu \mathbb{I})^{-1},
$$


therefore equation (4.9) is straigthforwardly satisfied.

Under the hypotheses listed at the beginning of this section (the phase space is the full space $\mathfrak{g l}(r)^{n}$, without constraints, and $A$ is suitably generic), the non constant coefficients of the characteristic equation are functionally independent and define exactly $\frac{1}{2} n r(r+1)$ hamiltonians. Fig. 1 displays the corresponding "fundamental molecule". One sees directly from the diagram that, for each one of the Poisson tensors $P_{0}, P_{1}$ and $P_{2}$, the set of hamiltonians $h_{i}^{j}$ includes exactly $n r$ Casimir functions, therefore the rank of the Poisson tensor cannot exceed $n r^{2}-n r=n r(r-1)$; on the other hand, the remaining $\frac{1}{2} n r(r-1)$ hamiltonians are in mutual involution, so the rank of the Poisson tensor cannot be less than $n r(r-1)$. Therefore, the fundamental Casimir polynomial $f_{\lambda \mu}$ is complete. If $A$ does not fulfill our requirements, what happens is that (i) the Poisson structures $P_{1}$ and $P_{2}$ have a larger kernel, so there are other independent Casimir functions not occurring in the characteristic determinant; (ii) some coefficients of the characteristic determinant vanish identically, and by consequence (iii) one cannot find properly normalised transversal vectorfields using the recipe presented below.

\subsection{Transversal vectorfields and separation coordinates}

The next step towards the construction of separation coordinates consists in finding a set of $P_{0}$-transversal vectorfields, normalized on the $n r$ Casimir functions which one gets from the fundamental Casimir polynomial. We state without proof the general recipe:

1. Choose a matrix $W_{1} \in \mathfrak{g l}(r)$ of rank one such that:

$$
\begin{aligned}
\operatorname{Tr}\left(W_{1}\right) & =0 \\
\operatorname{Tr}\left(W_{1} A\right) & =0 \\
& \vdots \\
\operatorname{Tr}\left(W_{1} A^{r-2}\right) & =0 \\
\operatorname{Tr}\left(W_{1} A^{r-1}\right) & =(-1)^{r-1} ;
\end{aligned}
$$

this condition makes sense because we know that $A^{k} \neq 0$ for $k<r$ (the $r$ linear equations above do not determine uniquely $W_{1}$, but any such $W_{1}$ will work).

2. Compute the adjoint of the characteristic matrix $L(\lambda)-\mu \mathbb{I}$, that we shall denote by $L^{\dagger}(\lambda, \mu)$; by definition, $(L(\lambda)-\mu \mathbb{I}) \cdot L^{\dagger}(\lambda, \mu)=f_{\lambda \mu} \mathbb{I}$. The entries of $L^{\dagger}(\lambda, \mu)$ are the cofactors of $L(\lambda)-\mu \mathbb{I}$, therefore $L^{\dagger}(\lambda, \mu)$ is polynomial of order $n(r-1)$ in $\lambda$ and of order $(r-1)$ in $\mu$.

3. Let $W_{\lambda}$ be the matrix polynomial of the form

$$
W_{\lambda}=W_{1} \lambda^{n-1}+W_{2} \lambda^{n-2}+\cdots+W_{n} .
$$


with

$$
\begin{aligned}
W_{2} & =W_{1}\left(u_{0,2} \mathbb{I}+u_{1,2} A+\cdots+u_{r-2,2} A^{r-2}\right) \\
& \vdots \\
W_{n} & =W_{1}\left(u_{0, n} \mathbb{I}+u_{1, n} A+\cdots+u_{r-2, n} A^{r-2}\right) .
\end{aligned}
$$

The $(r-1) \times(n-1)$ coefficients $u_{i, j}$ are scalar functions on $\mathfrak{g l}(r)^{n}$, which should be determined by the following condition: take the polynomial $\operatorname{Tr}\left(L^{\dagger}(\lambda, \mu) W_{\lambda}\right)$; for each power of $\mu$ separately, the $n$ highest coefficients in $\lambda$ should vanish, except for the coefficient of $\lambda^{n(r-1)+(n-1)}$, which is always equal to one because of (4.10). Namely, the terms which should be canceled with the appropriate choice of $u_{i, j}$ are the following:

$$
\begin{array}{crl}
\lambda^{k}, & n(r-1) & \leq k \leq n(r-1)+(n-2), \\
\mu \lambda^{k}, & (n-1)(r-1) & \leq k \leq(n-1)(r-1)+(n-1), \\
\vdots & 0 \leq k \leq(n-1)
\end{array}
$$

This gives a linear system of equations for the coefficients $u_{i, j}$. The system is triangular and can always be solved.

4. Denoting a tangent vector on $\mathfrak{g l}(r)^{n}$ by $\mathbf{v}=\left[\dot{M}_{1}, \ldots, \dot{M}_{n}\right]$, introduce the $n$ vectorfields

$$
\begin{aligned}
\mathbf{v}_{1}^{0} & =\left[0, \cdots, 0,0, W_{1}\right] \\
\mathbf{v}_{2}^{0} & =\left[0, \cdots, 0, W_{1}, W_{2}\right] \\
& \vdots \\
\mathbf{v}_{n}^{0} & =\left[W_{1}, \cdots, W_{n-1}, W_{n}\right]
\end{aligned}
$$

5. Take for $k=1, \ldots, r-1$ the product of $W_{\lambda}$ with the $k$-th power of the Lax matrix $L(\lambda)$ :

$$
W_{\lambda}^{(k)}=\frac{W_{\lambda} L^{k}}{\lambda^{n k}}
$$

Consider the highest $n$ terms in the expansion $W_{\lambda}^{(k)}=W_{1}^{(k)} \lambda^{n-1}+W_{2}^{(k)} \lambda^{n-2}+\ldots+$ $W_{n}^{(k)} \lambda^{0}+\ldots$ (in particular, one has $\left.W_{1}^{(k)} \equiv W_{1} A^{k}\right)$ : other $n(r-1)$ vectorfields $\mathbf{v}_{j}^{k}$, for $i=1 \ldots, n$ and $k=1, \ldots,(r-1)$, are defined analogously to (4.13):

$$
\begin{aligned}
\mathbf{v}_{1}^{k} & =\left[0, \cdots, 0,0, W_{1}^{(k)}\right] \\
\mathbf{v}_{2}^{k} & =\left[0, \cdots, 0, W_{1}^{(k)}, W_{2}^{(k)}\right] \\
& \vdots \\
\mathbf{v}_{n}^{k} & =\left[W_{1}^{(k)}, \cdots, W_{n-1}^{(k)}, W_{n}^{(k)}\right]
\end{aligned}
$$


For the $n r$ vectorfields constructed in this way, the following holds:

Proposition 4.12: The vectorfields $\mathbf{v}_{j}^{k}$ are symmetries of $P_{0}$ and fulfill the normalization condition

$$
\mathbf{v}_{j}^{k}\left(h_{m+(r-k-1) n}^{l}\right)=\delta^{k l} \delta_{j m} \quad \text { for } k, l=0, \ldots(r-1) \text { and } j, m=1, \ldots, n
$$

moreover, for all $k, j, l$ and $m$ in the given ranges,

$$
\mathbf{v}_{j}^{k}\left(\mathbf{v}_{m}^{l}\left(f_{\mu \nu}\right)\right)=0 \quad \text { and } \quad\left[\mathbf{v}_{j}^{k}, \mathbf{v}_{m}^{l}\right]=0
$$

To ensure that the $P_{0}$-transversal vectorfields $\mathbf{v}_{j}^{k}$ provide a set of (separation) DarbouxNijenhuis coordinates, one should also check conditions (2) and (3) of Prop.(3.7). However, this verification may be postponed: let us tentatively assume that these conditions are satisfied. Then, one could find separation coordinates in which the Poisson tensors $P_{0}, P_{1}$ and $P_{2}$ assume the "canonical" form described in section (3.3); in particular, the affine Lie-Poisson tensor $P_{0}$ becomes canonical in the usual sense.

In separation coordinates, we already know the explicit form of the $n r$ polynomials $S_{\alpha}(\lambda, \mu)$; the latter should coincide, up to the change of coordinates, with the polynomials $S_{j}^{k}(\lambda, \mu) \equiv \mathbf{v}_{j}^{k}\left(f_{\lambda \mu}\right)$ that one can also compute in terms of the original variables of the Lax equation. Hence, one should recover the separation coordinates by taking the common roots of the latter polynomials. Actually, it is sufficient to compute the common roots of the polynomials $\mathbf{v}_{1}^{k}\left(f_{\mu \nu}\right)$. The vectorfields $\mathbf{v}_{1}^{k}$ are constant: namely, we have seen that they are defined by $\dot{M}_{i}=0$ for $i=1, \ldots,(n-1)$ and $\dot{M}_{n}=W_{1} A^{k}$. The corresponding polynomials $S_{1}^{k}(\lambda, \mu)$ are the derivatives of the characteristic determinant $f_{\lambda \mu}$ along constant vectors fields, and therefore are nothing but linear combinations of cofactors of the characteristic matrix $L(\lambda)-\mu \mathbb{I}$, with constant coefficients determined by the matrices $W_{1}$ and $A$. Therefore, we recover Sklyanin's recipe, with a definite prescription of the normalization to be used 4

If one were able to compute $\frac{1}{2} n r(r-1)$ pairs of common roots $\left(\lambda_{i}, \mu_{i}\right)$ of the polynomials $S_{1}^{k}(\lambda, \mu)$, then one would only have to check a posteriori that the new variables $\left(\lambda_{i}, \mu_{i}\right)$ are canonical for the Poisson structure $P_{0}$. Since the polynomials $S_{1}^{k}(\lambda, \mu)$ are independent linear combinations of cofactors of the characteristic matrix, $\left(\lambda_{i}, \mu_{i}\right)$ are also roots of the fundamental Casimir polynomial, and this would be enough to say that all the hamiltonians become separable in the new coordinates. In this case there is no ambiguity on the constant part of $f_{\lambda \mu}$ itself, which turn out to depend only on the choice of $A$. Notice that in the new coordinates on $\mathfrak{g l}(r)^{n}$, given by the roots $\left(\lambda_{i}, \mu_{i}\right)$ and

\footnotetext{
${ }^{2}$ To be honest, exactly because Sklyanin's prescription does not fix the normalisation of the BakerAkhiezer function, to prove the equivalence of the two procedures one cannot rely on the simple comparison on the final results, but it would be necessary to compare each object involved in the two constructions, what we have not done yet.
} 
by the $n r$ Casimir functions of $P_{0}$, the vectorfields $\mathbf{v}_{j}^{k}$ automatically become coordinate vectorfields, due to the normalization and to the property (4.17). However, as we have stressed in the introduction, finding explicitly the roots of the polynomials $S_{1}^{k}(\lambda, \mu)$ is impossible in general.

A more effective method is the following one: since we know the expression of the polynomials $S_{j}^{k}(\lambda, \mu)$ in both coordinate systems, we can simply equate the corresponding coefficients for each polynomial to get a set of algebraic equations linking the two coordinate systems. Luckily enough, while the coefficients of $S_{j}^{k}(\lambda, \mu)$ are in general rather complicated rational functions of the separation coordinates, it is easy to see that a number of coefficients are linear functions of the entries of the Lax matrix $L(\lambda)$. It turns out that one can provide in this way a complete set of equations relating the two coordinate systems, which either are all linear (in the lower dimensional cases) or can be reduced to linear equations. In this way one can effectively compute the inverse coordinate transformation, i.e. express the original variables as functions of the separation coordinates. The mapping cannot be explicitly inverted in general, but it is sufficient for some purposes, for instance to check that the Poisson tensors transform as expected.

Let us present a concrete example. We have already displayed in sect.(3.3) the form of the polynomials $S_{\alpha}$ for the system associated to the Lie algebra $\mathfrak{g l}(3)$. Let us now compute the same polynomials for the Lax matrix $A \lambda+M$, with $M \in \mathfrak{g l}(3)$ and $A$ diagonal with distinct eigenvalues $(\alpha, \beta, \gamma)$ (this case corresponds to the generalised Euler-Poinsot rigid body, discussed in the introduction). We choose a set of orthonormal coordinates $\left(x_{1}, \ldots, x_{9}\right)$ in $\mathfrak{g l}(3)$, setting

$$
M=\left(\begin{array}{ccc}
x_{7} & \frac{1}{\sqrt{2}}\left(x_{1}+x_{4}\right) & \frac{1}{\sqrt{2}}\left(x_{2}+x_{5}\right) \\
\frac{1}{\sqrt{2}}\left(x_{4}-x_{1}\right) & x_{8} & \frac{1}{\sqrt{2}}\left(x_{3}+x_{6}\right) \\
\frac{1}{\sqrt{2}}\left(x_{5}-x_{2}\right) & \frac{1}{\sqrt{2}}\left(x_{6}-x_{3}\right) & x_{9}
\end{array}\right)
$$

The three Casimir functions for $P_{0}($ see $(1.27)$ ) are

$$
\begin{aligned}
h_{2}^{0} & =\beta \gamma x_{7}+\alpha \gamma x_{8}+\alpha \beta x_{9} \\
h_{1}^{1} & =-(\beta+\gamma) x_{7}-(\alpha+\gamma) x_{8}-(\alpha+\beta) x_{9} \\
h_{0}^{2} & =x_{7}+x_{8}+x_{9}
\end{aligned}
$$

Hence, there is a linear correspondence between the coordinates $\left(x_{7}, x_{8}, x_{9}\right)$ and the coordinates $\left(c_{1}, c_{2}, c_{3}\right)$. Choosing $W_{1}$ to have all the rows equal to each other, following step (1) above one finds

$$
W_{1}=\left(\begin{array}{lll}
\frac{1}{(\alpha-\beta)(\alpha-\gamma)} & \frac{1}{(\gamma-\beta)(\alpha-\beta)} & \frac{1}{(\beta-\gamma)(\alpha-\gamma)} \\
\frac{1}{(\alpha-\beta)(\alpha-\gamma)} & \frac{1}{(\gamma-\beta)(\alpha-\beta)} & \frac{1}{(\beta-\gamma)(\alpha-\gamma)} \\
\frac{1}{(\alpha-\beta)(\alpha-\gamma)} & \frac{1}{(\gamma-\beta)(\alpha-\beta)} & \frac{1}{(\beta-\gamma)(\alpha-\gamma)}
\end{array}\right)
$$


There are only three constant transversal vectorfields in this case, with $\dot{M}=W_{1}, \dot{M}=$ $W_{1} A$ and $\dot{M}=W_{1} A^{2}$ respectively. Taking the derivatives of $f_{\lambda \mu}=\operatorname{det}(A \lambda+M-$ $\mu \mathbb{I I})$ along these three vectorfields one easily computes the three polynomials $S_{1}(\lambda, \mu)$, $S_{2}(\lambda, \mu)$ and $S_{3}(\lambda, \mu)$. Let us write down only the coefficients which are useful for our purpose:

$$
\begin{aligned}
& S_{1}=\lambda^{2}+\frac{1}{\sqrt{2}}\left[-(\alpha-2 \gamma+\beta) x_{1}+(\alpha-2 \beta+\gamma) x_{2}+(2 \alpha-\beta-\gamma) x_{3}\right. \\
& +\sqrt{2}(\gamma-\beta) x_{7}+\sqrt{2}(\alpha-\gamma) x_{8}+\sqrt{2}(\beta-\alpha) x_{9} \\
& \left.+(\alpha-\beta) x_{4}-(\alpha-\gamma) x_{5}+(\beta-\gamma) x_{6}\right][(\gamma-\beta)(\alpha-\beta)(\alpha-\gamma)]^{-1} \mu \\
& +\frac{1}{\sqrt{2}}\left[\gamma(\alpha-2 \gamma+\beta) x_{1}-\beta(\alpha-2 \beta+\gamma) x_{2}-\alpha(2 \alpha-\beta-\gamma) x_{3}\right. \\
& +\sqrt{2}(\gamma-\beta)(-\gamma+\alpha-\beta) x_{7}-\sqrt{2}(\alpha-\gamma)(\alpha-\beta+\gamma) x_{8} \\
& +\sqrt{2}(\alpha-\beta)(\alpha-\gamma+\beta) x_{9}+\alpha(\gamma-\beta) x_{6}-\gamma(\alpha-\beta) x_{4} \\
& \left.+\beta(\alpha-\gamma) x_{5}\right][(\gamma-\beta)(\alpha-\beta)(\alpha-\gamma)]^{-1} \lambda+\ldots \\
& S_{2}=\lambda \mu+\frac{1}{\sqrt{2}}\left[(2 \alpha \gamma-\beta \gamma-\alpha \beta) x_{2}+(\alpha \gamma-2 \beta \gamma+\alpha \beta) x_{3}\right. \\
& +(-2 \alpha \beta+\beta \gamma+\alpha \gamma) x_{1}+\sqrt{2}(\alpha-\gamma) \beta x_{8}-\sqrt{2}(\alpha-\beta) \gamma x_{9} \\
& -\alpha(-\beta+\gamma) x_{6}+\sqrt{2} \alpha(-\beta+\gamma) x_{7}+\gamma(\alpha-\beta) x_{4} \\
& \left.-\beta(\alpha-\gamma) x_{5}\right][(\gamma-\beta)(\alpha-\beta)(\alpha-\gamma)]^{-1} \mu \\
& +\frac{1}{\sqrt{2}}\left[-\beta(2 \alpha \gamma-\beta \gamma-\alpha \beta) x_{2}\right. \\
& -\alpha(\alpha \gamma-2 \beta \gamma+\alpha \beta) x_{3}-\gamma(-2 \alpha \beta+\beta \gamma+\alpha \gamma) x_{1} \\
& -\sqrt{2} \alpha \gamma(\alpha-\gamma) x_{8}+\sqrt{2} \alpha \beta(\alpha-\beta) x_{9}+\alpha^{2}(-\beta+\gamma) x_{6} \\
& -\sqrt{2} \gamma \beta(-\beta+\gamma) x_{7}-\gamma^{2}(\alpha-\beta) x_{4} \\
& \left.+\beta^{2}(\alpha-\gamma) x_{5}\right][(\gamma-\beta)(\alpha-\beta)(\alpha-\gamma)]^{-1} \lambda+\ldots \\
& S_{3}=\lambda^{2}+\frac{1}{\sqrt{2}}\left[\left(\gamma^{2} \alpha-\beta \gamma^{2}-\alpha^{2} \beta+\alpha^{2} \gamma\right) x_{2}+\left(\gamma^{2} \alpha-\beta \gamma^{2}+\beta^{2} \alpha-\beta^{2} \gamma\right) x_{3}\right. \\
& +\left(-\beta^{2} \alpha+\beta^{2} \gamma-\alpha^{2} \beta+\alpha^{2} \gamma\right) x_{1}-\sqrt{2}(-\alpha \beta+\alpha \gamma-\beta \gamma)(\alpha-\gamma) x_{8} \\
& -\sqrt{2}(-\alpha \beta+\alpha \gamma+\beta \gamma)(\alpha-\beta) x_{9}-(\alpha \gamma-\beta \gamma+\alpha \beta)(-\beta+\gamma) x_{6} \\
& +\sqrt{2}(\alpha \gamma-\beta \gamma+\alpha \beta)(-\beta+\gamma) x_{7}+(-\alpha \beta+\alpha \gamma+\beta \gamma)(\alpha-\beta) x_{4} \\
& \left.+(-\alpha \beta+\alpha \gamma-\beta \gamma)(\alpha-\gamma) x_{5}\right] \text {. } \\
& \cdot[(\gamma-\beta)(\alpha-\beta)(\alpha-\gamma)]^{-1} \mu
\end{aligned}
$$




$$
\begin{aligned}
& +\frac{1}{\sqrt{2}}\left[-\gamma\left(-\beta^{2} \alpha+\beta^{2} \gamma-\alpha^{2} \beta+\alpha^{2} \gamma\right) x_{1}-\sqrt{2} \alpha \gamma \beta(-\beta+\gamma) x_{7}\right. \\
& -\beta\left(\gamma^{2} \alpha-\beta \gamma^{2}-\alpha^{2} \beta+\alpha^{2} \gamma\right) x_{2}-\sqrt{2} \alpha \beta \gamma(\alpha-\gamma) x_{8} \\
& -\alpha\left(\gamma^{2} \alpha-\beta \gamma^{2}+\beta^{2} \alpha-\beta^{2} \gamma\right) x_{3}+\sqrt{2} \alpha \beta \gamma(\alpha-\beta) x_{9} \\
& -(-\alpha \beta+\alpha \gamma+\beta \gamma)(\alpha-\beta) \gamma x_{4} \\
& \left.-(-\alpha \beta+\alpha \gamma-\beta \gamma)(\alpha-\gamma) \beta x_{5}\right] \\
& +(\alpha \gamma-\beta \gamma+\alpha \beta)(-\beta+\gamma) \alpha x_{6} \\
& \cdot[(\gamma-\beta)(\alpha-\beta)(\alpha-\gamma)]^{-1} \lambda+\ldots
\end{aligned}
$$

Therefore, there are exactly six coefficients which are linear in the coordinates $x_{i}$. One can check that they are independent and also independent from the other three equations already found from (4.19): the determinant of the full $9 \times 9$ linear system is identically equal to one. By equating the polynomials above to the corresponding expressions listed at the end of sect.(3.3), one finds the coordinate transformation. On the other hand, all three polynomials contain quadratic terms in $\lambda$ and $\mu$, and to find the common roots of two of them one should solve an equation of order four in $\lambda$ (or in $\mu$ ), which is possible in principle but gives a result which is of little practical use.

The generalization of the Lagrange top can be treated in the same way. The phase space is $\mathfrak{g l}(3)^{2}$, and as above we use as coordinates suitable orthonormal linear combinations of the entries of $M_{1}$ and $M_{2}$, that we denote by $x_{i}, i=1, \ldots, 18$. The constant matrix for this case is chosen to be $A=\left(\begin{array}{ccc}0 & 1 & 0 \\ -1 & 0 & 0 \\ 0 & 0 & 0\end{array}\right)$. The Casimir functions for $P_{0}$ occurring in the characteristic determinant $f_{\lambda \mu}=\operatorname{det}\left(A \lambda^{2}+M_{1} \lambda+M_{2}\right)$ are $h_{4}^{0}, h_{5}^{0}, h_{2}^{1}$, $h_{3}^{1}, h_{0}^{2}$ and $h_{1}^{2}$; they are linear except for $h_{4}^{0}$ and $h_{2}^{1}$ which are quadratic. There are 6 transversal vectorfields, three of which are constant. Each of the three corresponding polynomials, $S_{4}^{0}(\lambda, \mu), S_{2}^{1}(\lambda, \mu)$ and $S_{0}^{2}(\lambda, \mu)$, has exactly three coefficients which are linear in the coordinates $x_{i}$, namely the coefficients of $\mu, \lambda \mu$ and $\lambda^{3}$. Then, one has a total of 13 independent linear equations which can be solved for the 13 coordinates $x_{1}, \ldots, x_{9}, x_{15}, \ldots, x_{18}$. The coefficients of $\lambda$ and $\lambda^{2}$ in the polynomials $S_{4}^{0}, S_{2}^{1}$ and $S_{0}^{2}$, are linear in the remaining five coordinates $x_{10}, \ldots, x_{14}$, which can thus be computed as well. On the contrary, the direct procedure à la Sklyanin, i.e. computing the common roots of the bivariate polynomials $S_{\alpha}$, is not viable due to the order of the polynomials themselves.

\section{Acknowledgements}

We are much indebted to Franco Magri, Gregorio Falqui, Marco Pedroni and Sergio Benenti for several helpful discussions and exchanges of information on work in progress, and to the referee for useful comments. This work is supported by the national MURST research project "Geometry of Integrable Systems". 


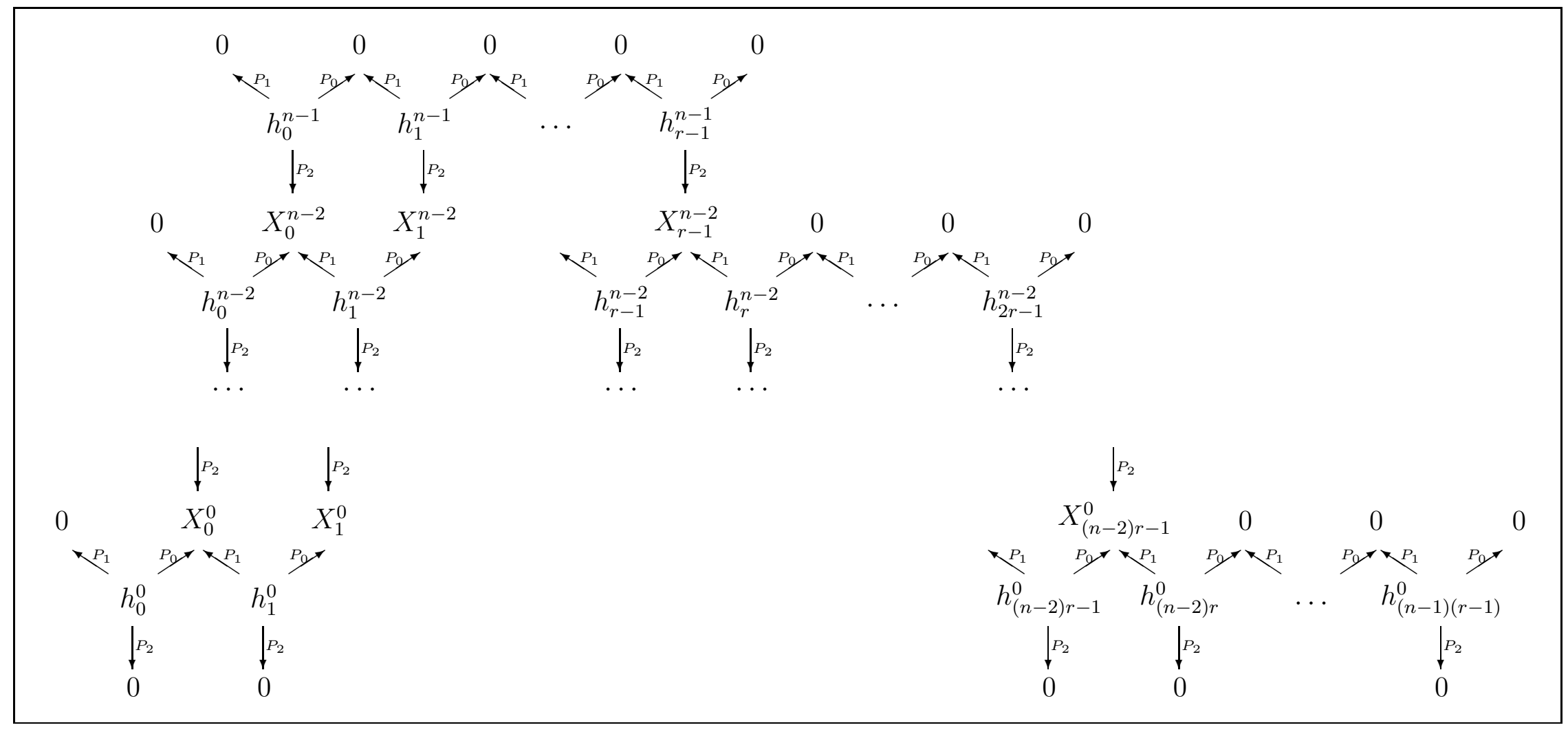

Figure 1: Fundamental molecule for $\mathfrak{g l}(r)^{n}$ 


\section{References}

[1] F. Magri, A Simple Model of the Integrable Hamiltonian Equation, J. Math. Phys., 19 (1978), 1156-1162.

[2] G. Falqui, F. Magri, M. Pedroni, in preparation.

[3] G. Falqui, M. Pedroni, Separation of variables for bi-Hamiltonian systems, nlin.SI/0204029.

[4] F. Magri, C. Morosi, A Geometrical Characterization of integrable Hamiltonian Systems through the Theory of Poisson-Nijenhuis Manifolds, Quaderno S 19/1984 of the Departement of Mathematics of the University of Milano, unpublished.

[5] F. Magri, Y. Kosmann-Schwarzbach, Lax-Nijenhuis Operators for Integrable Systems, J. Math. Phys., 37 (1996), 6173-6197.

[6] P. Casati, F. Magri, M. Pedroni [1992], Bi-hamiltonian Manifolds and $\tau$ function, in: Mathematical Aspects of Classical Field Theory (1991), M.J. Gotay et al. eds., Contemporary Mathematics, 132, American Mathematical Society, Providence.

[7] T. MARsico, Una caratterizzazione geometrica dei sistemi che ammettono rappresentazione alla Lax estesa, unpublished PhD Thesis, University of Milano (1995).

[8] M.Adler, P. VAn Moerbeke, Completely integrable systems, Euclidean Lie algebras, and curves, Adv. in Math. 38 (1980), 267-317.

M.Adler, P. VAN MoERBEKe, Linearization of Hamiltonian systems, Jacobi varieties and representation theory, Adv. in Math. 38 (1980), 318-379.

[9] P.A. Griffiths, Linearizing Flows and Cohomological Interpretation of Lax Equations, Amer. J. of Math., 107 (1985), 1445-1483.

[10] G. Magnano, F. Magri, Poisson-Nijenhuis Structures and Sato Hierarchy, Rev. Math. Phys., 3 (1991), 403-466.

[11] G. Magnano, Bihamiltonian Approach to Lax Equations with Spectral Parameter, Acc. Sc. Torino Mem. Sc. Fis., 19-20 (1995-1996), 159-209.

[12] E. Sklyanin, Separations of Variables. New Trends, Progr. Theo.r Phys. Suppl., 118 (1995), 35-60.

[13] S.V. Manakov, Note on the Integration of Euler's Equation of the Dynamics of an n-dimensinal Rigid Body, Funkt. Anal. Appl., 10 (1976), 93-94. 
[14] M. Ugaglia, Sistemi dinamici su algebre di Lie: funzioni di Casimir e significato del parametro spettrale, unpublished BSc Thesis, University of Torino (1994), and private communication.

[15] C. Morosi, L. Pizzocchero, On the Euler Equation: Bi-hamiltonian Structures and Integrals in Involution, Lett. Math. Phys., 37 (1996), 117-135.

[16] C. Morosi, L. Pizzocchero, r-matrix Theory, Formal Casimirs and the Periodic Toda Lattice, J. Math. Phys., 37 (1996), 4484-4513.

[17] T. RAtiu, Euler-Poisson Equations on Lie Algebras and the n-dimensional Heavy Rigid Body, American J. Math., 104 (1982), 409-448.

[18] G. Falqui, F. Magri, M. Pedroni, Bihamiltonian Geometry, Darboux Coverings and Linearization of the KP Hierarchy, Comm. Math. Phys., 197 (1998), 303324 .

[19] W. Oevel, O. Ragnisco, R-matrices and Higher Poisson Brackets for Integrable Systems, Phys. A, 161 (1989), 181-220.

[20] M. A. Semënov-Tyan-Shansky, What is a Classical R-Matrix?, Funct. Anal. Appl., 17 (1983), 259-272.

[21] A. G. Reyman, M. A. Semënov-Tyan-Shansky, Compatible Poisson structures for Lax equations: an r-matrix approach, Phys. Lett. A, 130 (1988), 456-460.

[22] A. G. Reyman, M. A. Semënov-Tyan-Shansky, Group-Theoretical Methods in the Theory of Finite-Dimensinal Integrable Systems, in: Encyclopaedia of Mathematical Sciences, V.I. Arnold and S.P. Novikov eds., 16, 116-225, Springer-Verlag, Berlin 1994.

[23] J. HARnAD, Loop groups, R-matrices and separation of variables. Integrable systems: from classical to quantum (Montréal, QC, 1999), 21-54, CRM Proc. Lecture Notes, 26, Amer. Math. Soc., Providence 2000.

[24] G. Falqui, F. Magri, M. Pedroni, J.P. Zubelli, A Bi-Hamiltonian Theory for Stationary KdV Flows and their Separability, Reg. Chaotic Dyn. 5 (2000), 33-52.

[25] L. Degiovanni, F. Magri, V. Sciacca, On deformation of Poisson manifolds of hydrodynamic type, preprint n. 127 of the Departement of Mathematics and Applications of the University of Palermo, submitted for publication. 